\title{
Demonstration of slow extraction loss reduction with the application of octupoles at the CERN Super Proton Synchrotron
}

\author{
Matthew Alexander Fraser $\odot$, Brennan Goddard, Verena Kain $\odot$, \\ Michelangelo Pari@, and Francesco Maria Velotti@ \\ CERN, CH-1211, Geneva 23, Switzerland \\ Linda Susanne Stoel@ and Michael Benedikt \\ CERN, CH-1211, Geneva 23, Switzerland and TU Wien, Karlsplatz 13, 1040 Vienna, Austria
}

(Received 20 August 2019; published 11 December 2019)

\begin{abstract}
The powering of octupoles during third-integer resonant slow extraction has been studied and recently tested with the beam at the CERN Super Proton Synchrotron (SPS) in order to increase the extraction efficiency and reduce the induced radioactivity along the extraction straight. The octupoles distort the particle trajectories in phase space in such a way that the extracted separatrix is folded, which decreases the particle density impinging the wires of the extraction septum at the expense of increasing the extracted beam emittance. During experimental SPS machine studies a reduction of over $40 \%$ in the specific (per extracted proton) beam loss measured at the extraction septum was achieved. In this paper, the prerequisite studies needed to safely deploy the new extraction scheme in a limited time-frame are described, the experimental results are presented and an outlook given toward the next steps to bring slow extraction with octupoles into routine operation.
\end{abstract}

DOI: 10.1103/PhysRevAccelBeams.22.123501

\section{INTRODUCTION}

Slow extraction using the third-integer resonance and thin electrostatic septa is a process with inherent beam loss, inducing radioactivation of the machine, reduced component lifetime, and severe limitations on personnel access and maintenance. In view of tightening restrictions on doseto-personnel for the necessary hands-on maintenance of accelerator equipment, and ever-increasing experimental requests for higher slow-extracted proton flux, the SPS Losses and Activation Working Group [1,2] was established to investigate, implement, and follow-up various methods to reduce the induced radioactivity in the extraction straight located in Long Straight Section 2 (LSS2) and the SPS in general. The flux of $400 \mathrm{GeV} / c$ protons delivered to the present experiments, located in the North Area of the SPS, is increasing year-on-year with a historical record of more than $1.2 \times 10^{19}$ protons on target last year. Future experimental proposals request a severalfold increase upwards of $2.0 \times 10^{20}$ protons on target over a timescale of 5 years, such as SHiP [3] at the proposed SPS Beam Dump Facility (BDF) [4].

*mfraser@cern.ch

Published by the American Physical Society under the terms of the Creative Commons Attribution 4.0 International license. Further distribution of this work must maintain attribution to the author(s) and the published article's title, journal citation, and DOI.
The application of higher-order multipole fields to manipulate the spatial density of the beam presented to the extraction septum is not a new concept and has been reported various times in literature [5-7]. It is one of the main slow extraction beam loss reduction techniques pursued at CERN in recent years [8-11]. In the present operational scenario four extraction sextupoles (LSE) are used to drive the third-integer resonance and increase the amplitude of particles on outward spiraling separatrices, which closely resemble straight lines in phase space. In this scenario, the spatial density of the beam at the septum drops off quadratically with amplitude, i.e., $\propto 1 / x^{2}$. When strong higher-order multipole fields are added, one can curve the phase space separatrix presented to the septum and manipulate it such that, after optimization and at the expense of a larger extracted beam emittance, the spatial density projection of the extracted beam is peaked inside the extraction channel rather than at the septum blade. The lower density at the septum blade reduces the number of protons interacting with it during extraction, reducing the overall beam loss and induced radioactivation. The essential goal of the scheme is to increase the sextupole strength whilst folding the beam back with the higher-order fields to avoid the extracted beam from striking the aperture limitation posed by the cathode of the electrostatic extraction septum. A full discussion on the technique of applying high-order multipoles, including both octupoles and decapoles, to third-integer slow extraction can be found in [11]. 
TABLE I. Machine parameters for slow extraction at SPS.

\begin{tabular}{lcc}
\hline \hline Parameter & Unit & Value \\
\hline Particle & $\mathrm{p}$ & \\
Nominal spill intensity & $10^{13}$ & $3-4$ \\
Spill length & $\mathrm{s}$ & $\sim 1-10$ \\
Momentum $\left(p_{0}\right)$ & $\mathrm{GeV} / c$ & 400 \\
Relativistic factor $\left(\beta_{r} \gamma_{r}\right)$ & & 426 \\
Magnetic rigidity $(B \rho)$ & $\mathrm{Tm}$ & 1334 \\
Momentum spread $\left(\Delta p / p_{0}\right)$ & $\%$ orm. $)$ & $-1.5,1.5]$ \\
Horizontal emittance $\left(\mathrm{rms}\right.$, norm. $\left(\varepsilon_{x, n}\right)$ & $\mu \mathrm{m}$ & 8 \\
Horizontal emittance $(\mathrm{rms}, \mathrm{geom}).\left(\varepsilon_{x, g}\right)$ & $\mathrm{nm}$ & 19 \\
Vertical emittance $(\mathrm{rms}$, norm. $)\left(\varepsilon_{y, n}\right)$ & $\mu \mathrm{m}$ & 5 \\
Vertical emittance $(\mathrm{rms}$, geom. $)\left(\varepsilon_{y, g}\right)$ & $\mathrm{nm}$ & 12 \\
Horizontal tune $\left(\right.$ on resonance) $\left(Q_{x}\right)$ & & $80 / 3$ \\
Vertical tune $\left(Q_{y}\right)$ & & 26.58 \\
Horizontal chromaticity $\left(\xi_{x}=Q_{x}^{\prime} / Q_{x}\right)$ & & -1.0 \\
Vertical chromaticity $\left(\xi_{y}=Q_{y}^{\prime} / Q_{y}\right)$ & & 0.5 \\
\hline \hline
\end{tabular}

\section{SLOW EXTRACTION AT THE SPS}

The nominal SPS slow extraction scheme is a momentum dominated, chromatic extraction driven by a tune sweep and characterized by a small transverse emittance. The nominal parameters for the SPS are collected in Table I. Once the beam arrives at flat-top with a momentum of $400 \mathrm{GeV} / c$ the momentum spread is enhanced with a radio-frequency (rf) manipulation before turning off the $\mathrm{rf}$ system ( $\pi$ phase jump on to the unstable phase followed by a bunch rotation on the stable phase). The increased momentum spread translates through a large chromaticity to a large chromatic tune spread. The machine tune starts below resonance and the extraction is driven by sweeping the tune up through the intrinsic tune spread of the beam by changing the current in the main quadrupole circuits. Since the chromaticity is negative, particles with low momenta have a higher tune, enter resonance first and are extracted earlier.

The layout and equipment of the slow extraction system located in LSS2 is shown in Fig. 1. The septa deflect the beam from the synchrotron into the transfer line headed toward the experiments located in the North Area. The first septum is a thin-wire electrostatic septum (ZS) made of 5 separate units, each $3.15 \mathrm{~m}$ in length and composed of an array of 2080 tungsten-rhenium wires strung on the anode support, which are individually aligned to the beam. The ZS is followed by a thin magnetic septum (MST) and a thick magnetic septum (MSE). The ZS is aligned such that the upstream end of the wire-array delimiting the extracted beam from the circulating beam is nominally located at an amplitude of $x_{\mathrm{ZS}}=68 \mathrm{~mm}$, representing the vertical axis of Fig. 1(b). Two absorbers are placed in the extraction region: the TCE is a cooled cast-iron mask, which protects the enlarged-aperture quadrupole QDA.217 directly after the ZS from stray particles that scatter or shower from the septum wires, and the TPST consists of aluminium and copper absorbers protecting the MST septum blade and its extraction aperture from such particles. After being deflected by the MSE the extracted beam passes through a window cut into the coil of QDA.219 to leave the synchrotron.

The size of the extracted beam in the horizontal plane is trimmed by adjusting the distance between the extraction septum and the circulating beam using the extraction bump amplitude and the LSE strength, see Eq. (8) and the discussion in Sec. VIB. The spiral step is adjusted to a value of approximately $15 \mathrm{~mm}$ to fit comfortably inside the gap between the anode (wire-array) and the cathode of

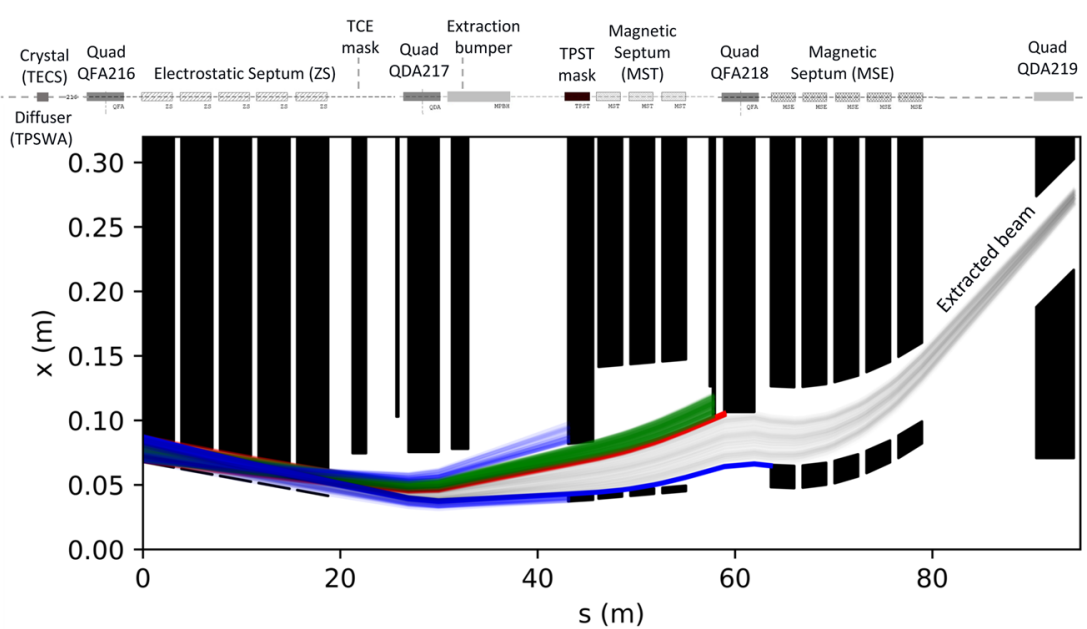

(a) Acceptance of extraction channel (grey tracks) limited by the aperture of wide-aperture quadrupole (QFA.218) and its upstream beam position monitor (BPCE.218)

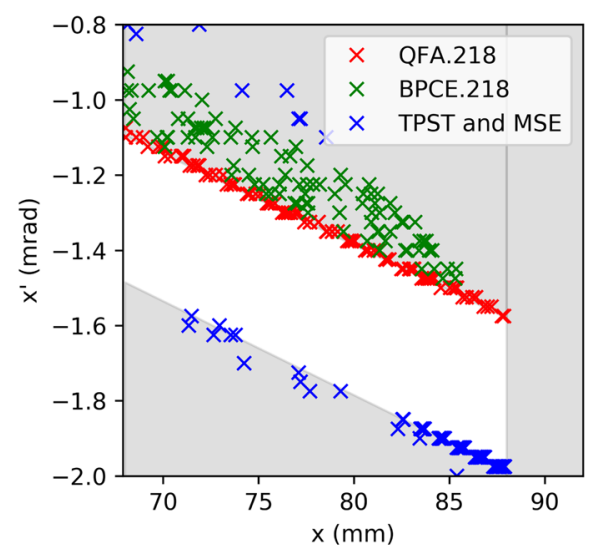

(b) Acceptance (white area) of the extraction channel at the upstream end on the high-field side of the ZS

FIG. 1. Layout of the LSS2 extraction straight and the phase space acceptance of the extraction channel at the entrance to the electrostatic septum (ZS). The phase space acceptance is delimited along with selected particle tracks lost on relevant apertures. The blade of the ZS cuts the circulating beam at an amplitude $x_{\mathrm{ZS}}=68 \mathrm{~mm}$. 
the ZS over which the high voltage is applied. The four LSE magnets each deliver a nominal integrated and normalized sextupole field of $k_{2 \text {, nom }} L=0.089 \mathrm{~m}^{-2}$.

The apertures in the horizontal plane along the extraction channel are presented in Fig. 1. The phase space acceptance at the upstream end of the ZS is shown schematically in Fig. 1(b) along with selected particles that are tracked to various relevant aperture restrictions. The color-coding shows the limiting apertures of various downstream devices. The enlarged horizontal emittance in the presence of phase space folding from applying strong octupole fields must fit inside the white shaded area of phase space, otherwise beam loss will occur.

\section{OCTUPOLES IN THE SPS}

The large number of octupoles installed in the SPS for the mitigation of transverse instabilities through Landau damping make it an ideal test-bed for applying higher-order fields to resonant slow extraction. The SPS ring has an approximate sixfold symmetry with each $64 \mathrm{~m}$ long sextant consisting of 18 FODO cells. Each half-cell contains four dipoles, with the exception of those in the LSS insertion regions located in the centre of each sextant. Each LSS consists of two FODO cells without any main dipoles, with a missing-magnet dispersion suppressor of one cell on each side.

The SPS is equipped with two Landau octupole families (LOF_-focusing and LOD_-defocusing) with each magnet capable of delivering an integrated, normalized octupole field of more than $k_{3} L=6 \mathrm{~m}^{-3}$. The LOF magnets are installed in locations of high dispersion near focusing quadrupoles where the horizontal Twiss function $\left(\beta_{x}\right)$ is large and typically $85-105 \mathrm{~m}$. The relevant optics functions at the locations of the octupoles in the lattice are summarized in Fig. 2. Only the LOF family were considered because they are an order of magnitude more effective than the LOD family, which are instead located near defocusing quadrupoles where $\beta_{x}$ is much smaller.

There are 24 LOF magnets in the machine powered by a single bipolar power converter in series and, even though 6 are cabled with an inverted polarity, there is adequate strength to exploit the parameter space required within the scope of the extraction studies presented in this paper.

The LOF and LOD families are not usually powered during extraction on the flat-top. In the measurements and simulations presented here, the LOF octupoles are turned on just before and held at a fixed normalized strength throughout the flat-top.

\section{A. Constant optics slow extraction (COSE)}

The constant optics slow extraction (COSE) technique, discussed in detail elsewhere [13], was employed to suppress the optics mismatch induced by the chromatic tune sweep scheme at the SPS. This was a prerequisite for

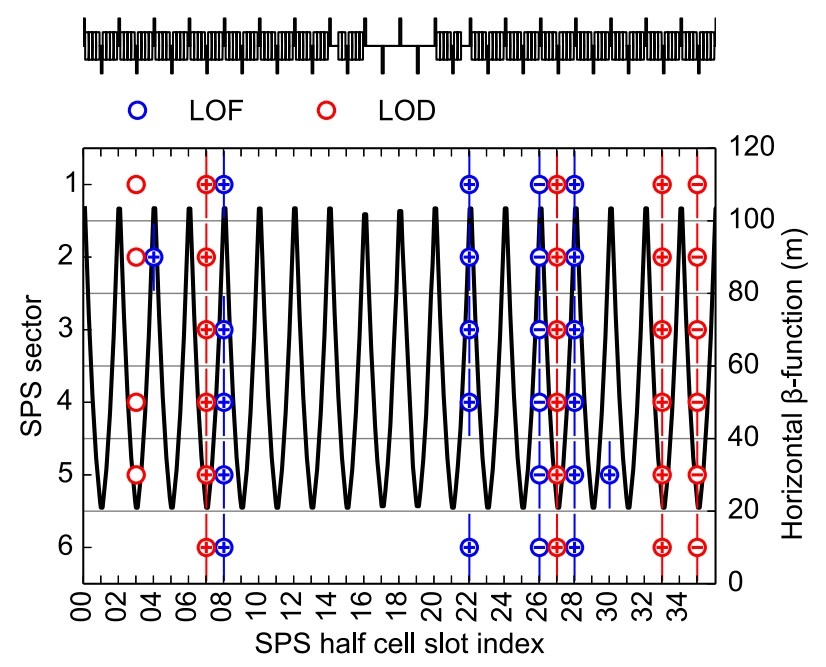

(a) Horizontal $\beta$-function, $\beta_{x}$

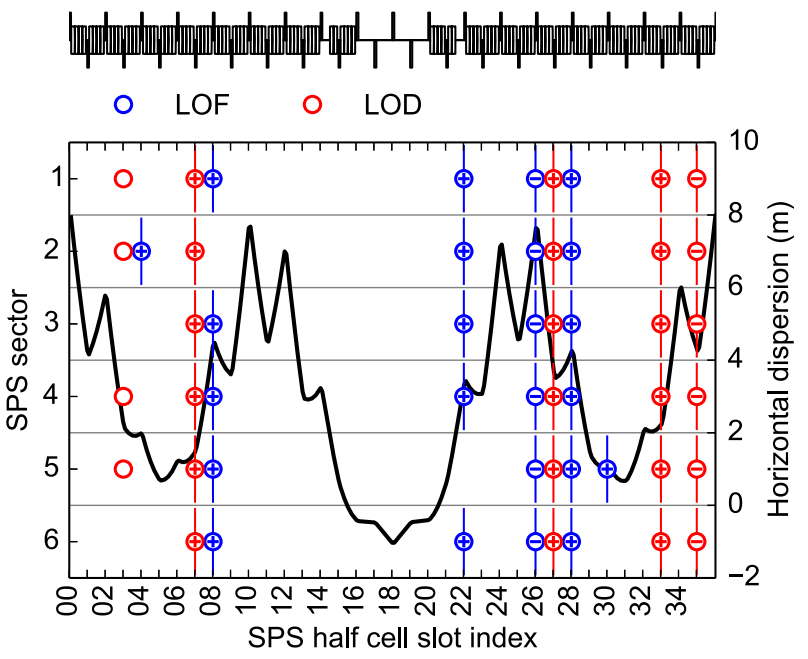

(b) Horizontal dispersion function, $D_{x}$

FIG. 2. Octupole locations and relevant optics functions in the SPS lattice per sector after recent modifications to the LOF and LOD powering configuration [12].

slow extraction with octupoles. COSE ramps the strength of all magnetic circuits synchronously, e.g., sextupoles, octupoles, extraction bump, magnetic extraction septa, etc., along with the main quadrupole circuit driving the tune sweep, in order to follow the changing momenta of the extracted particles throughout the spill. COSE suppresses the emittance blow-up during extraction in both planes that arises from the optical mismatch and, particularly in the case of octupoles, the effects of dispersion in the LOF magnets.

\section{B. The dispersion problem in the SPS}

The variation of the extracted beam momentum couples with the large dispersion ( 3-7 $\mathrm{m}$ ) at the LOF octupoles to blow-up the extracted beam emittance when time-averaged 


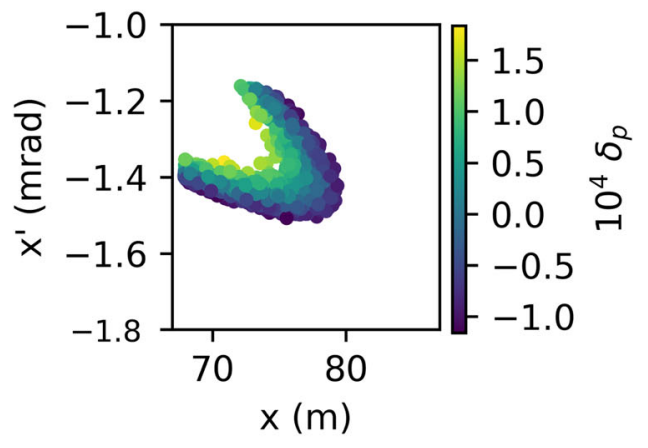

(a) $t=115 \mathrm{~ms}$

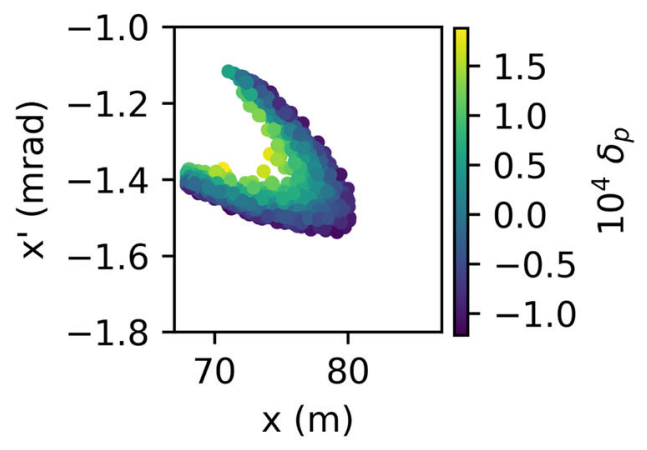

(b) $t=345 \mathrm{~ms}$

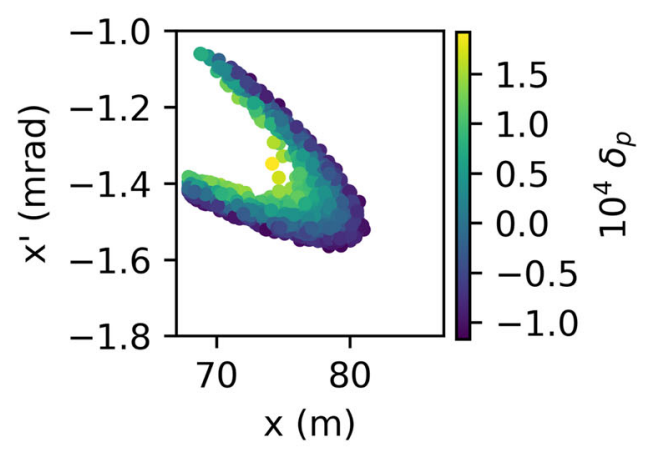

(c) $t=575 \mathrm{~ms}$

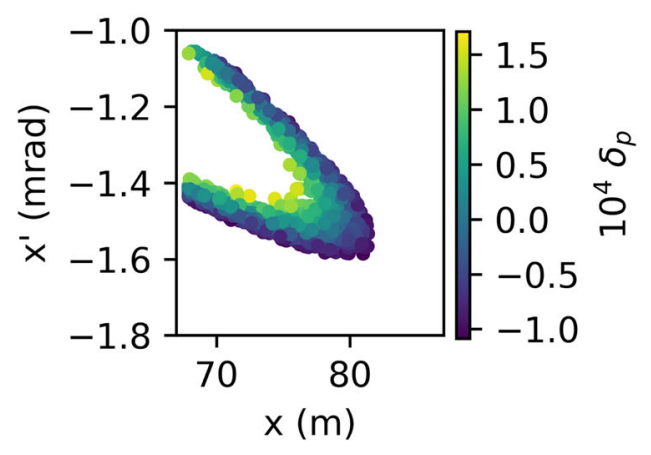

(d) $t=805 \mathrm{~ms}$

FIG. 3. $Q$-sweep (time dependent): instantaneous phase space presentation to the high-field side of the ZS in the presence of octupole fields simulated during a short spill on a $1.2 \mathrm{~s}$ flat-top.

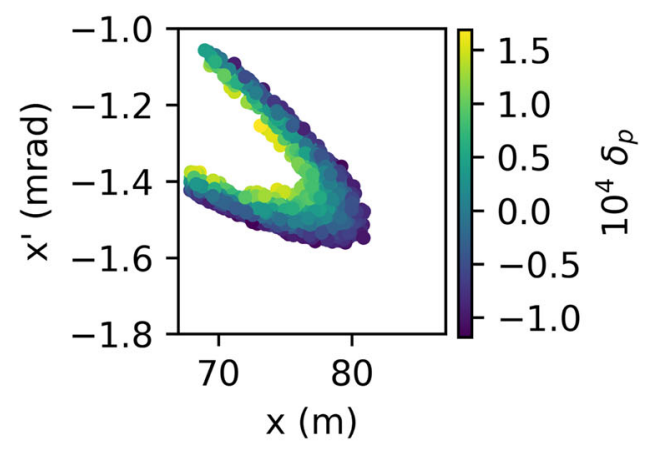

(a) $t=115 \mathrm{~ms}$

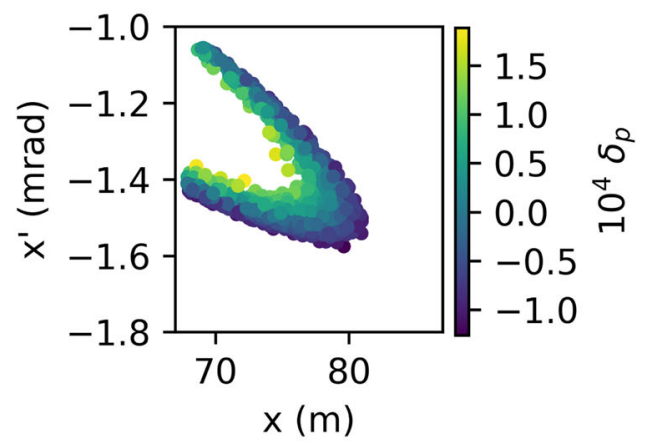

(b) $t=345 \mathrm{~ms}$

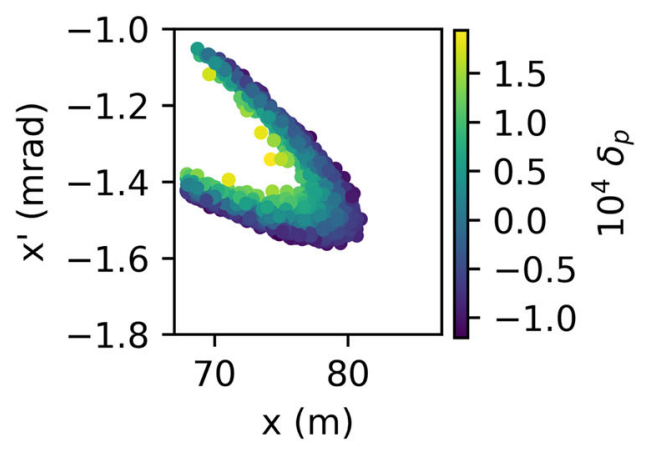

(c) $t=575 \mathrm{~ms}$

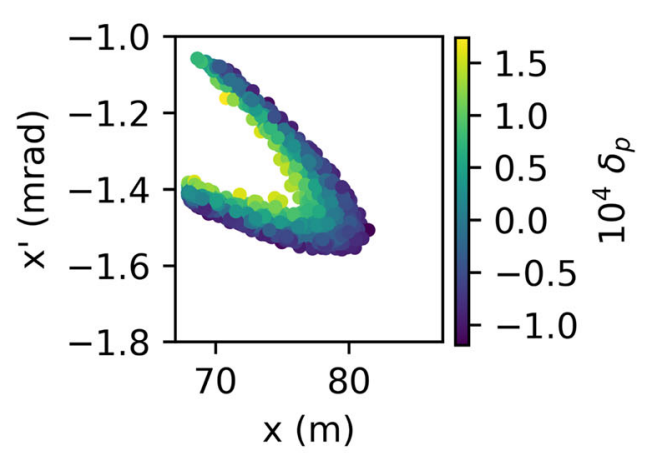

(d) $t=805 \mathrm{~ms}$

FIG. 4. COSE (time independent): instantaneous phase space presentation to the high-field side of the ZS in the presence of octupole fields simulated during a short spill on a $1.2 \mathrm{~s}$ flat-top. 
over the spill. The dispersive steering of the on-resonance particles (being extracted at any given instance of time) in the octupoles creates a phase space folding that is timedependent, as illustrated by the simulations presented in Fig. 3. Even more concerning is the variation of the angle at which the beam strikes the wire-array of the ZS throughout the spill and the resulting increase in beam loss, which in some cases nullifies the reduction in beam density achieved with the application of the octupoles. As shown for comparison in Fig. 4, the time-dependent effect is suppressed when COSE is applied. This is achieved because the COSE scheme includes a ramp function on the current

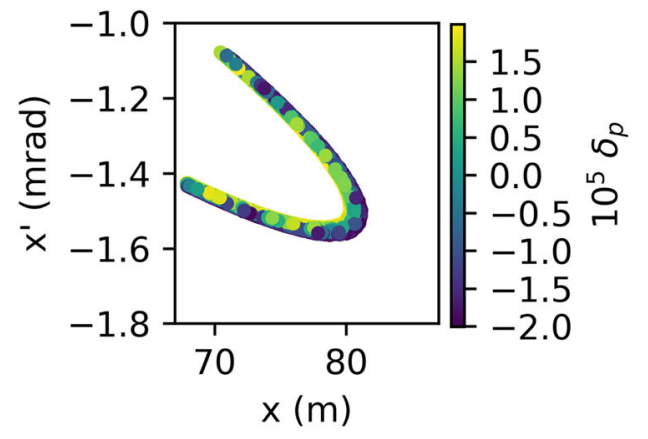

(a) $\sigma_{\delta_{p}}=1 \times 10^{-4}$

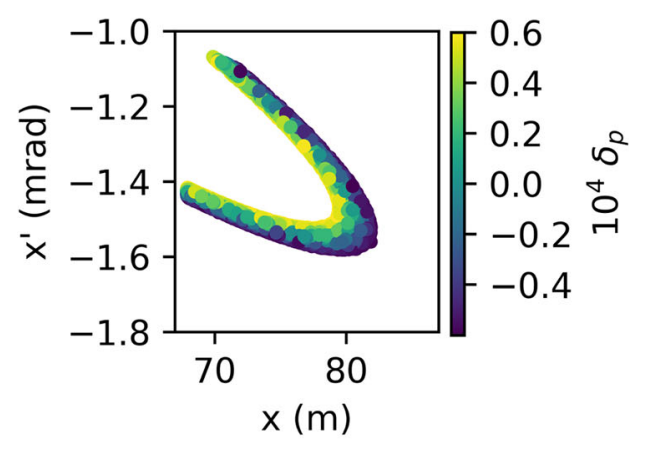

(b) $\sigma_{\delta_{p}}=3 \times 10^{-4}$

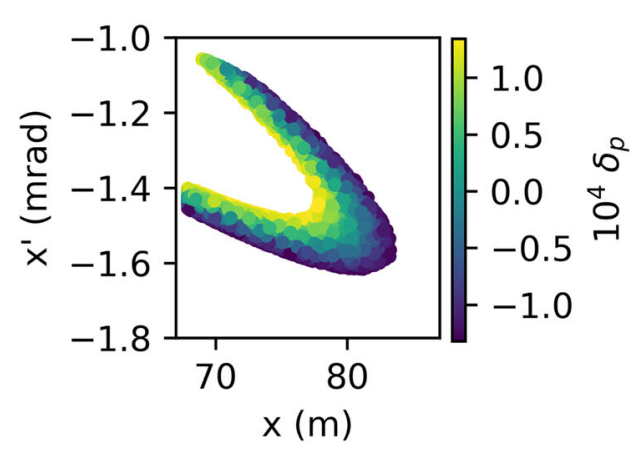

(c) $\sigma_{\delta_{p}}=6 \times 10^{-4}$

FIG. 5. Impact of the instantaneous momentum spread $\left(\sigma_{\delta_{p}}\right)$ (proportional to the resonance stop-band width) on the instantaneous phase space presentation of the beam to the high-field side of the ZS. in the main dipole circuit that follows the extracted beam momentum. As a result, the particles that are flowing along the separatrices of the third-integer resonance at any instance in time during the extraction are held at the same position inside the LOF magnets regardless of their momentum.

A significant drawback of applying octupoles located in regions of high dispersion, as is the case at the SPS, is the unavoidable blow-up of the extracted emittance caused by the intrinsic momentum spread, in addition to the folding. Although COSE removes the time-dependence caused by the tune sweep, it cannot compensate chromatic effects driven by the intrinsic momentum spread. This is especially the case as the stop-band width of the resonance increases when the sextuple strength is increased. Particles entering the octupoles on a dispersive orbit see a field strength correlated to their momentum and the amount by which they are folded varies. This is illustrated by the correlation with momentum present in Figs. 3 and 4. The additional emittance blow-up arising from this effect is shown in Fig. 5 with simulations carried out at a fixed instance in time and with an artificially modified instantaneous momentum distribution, approximated by a Gaussian distribution and parametrized by its variance $\left(\sigma_{\delta_{p}}^{2}\right)$.

\section{KOBAYASHI HAMILTONIAN WITH OCTUPOLES}

A modern derivation of the Kobayashi Hamiltonian, which is traditionally used to describe the dynamics of charged particles close to the third-order resonance, can be found in [14], however this derivation only considers sextupoles. The original paper [15] does treat arbitrarily high multipoles, but the notation used is rather far removed from today's conventions. Assuming a single multipole magnet of length $L$ with arbitrary normalized multipole components,

$$
k_{n}=\frac{1}{B \rho}\left[\frac{\partial^{n} B_{y}}{\partial x^{n}}\right]_{x=y=0},
$$

or several multipoles that can be approximated as a single effective multipole, one can express the normalized and integrated multipole strength as,

$$
K_{n}=\frac{k_{n} L}{n !} \beta_{x}^{(n+1) / 2}
$$

where the multipole is situated at a location in the lattice with $\beta_{x}$. Using nondimensionalized coordinates, assuming $K_{2} \neq 0$, the Hamiltonian can be written with an additional term in the case of octupoles as, 
$\mathcal{H}=\frac{\varepsilon}{2}\left(\hat{X}^{2}+\hat{P}^{2}\right)+\frac{1}{4}\left(\hat{X}^{3}-3 \hat{X} \hat{P}^{2}\right)+\frac{9}{32} \kappa_{3}\left(\hat{X}^{2}+\hat{P}^{2}\right)^{2}$

where $X$ and $P$ are the normalized phase space coordinates reexpressed in nondimensionalized coordinates as,

$$
(\hat{X}, \hat{P})=K_{2} \cdot(X, P),
$$

$\kappa_{3}=K_{3} / K_{2}^{2}$ and the tune distance from resonance is $\varepsilon=6 \pi\left(Q-Q_{\text {res }}\right)$. The threefold rotational symmetry of the Hamiltonian is easier to notice in polar coordinates $(\hat{A}, \hat{\theta})$ defined by,

$$
\hat{X}=\hat{A} \cos \hat{\theta} \quad \text { and } \quad \hat{P}=\hat{A} \sin \hat{\theta},
$$

with the Hamiltonian equivalently expressed as,

$$
\mathcal{H}=\frac{\varepsilon}{2} \hat{A}^{2}+\frac{1}{4} \hat{A}^{3} \cos 3 \hat{\theta}+\frac{9}{32} \kappa_{3} \hat{A}^{4} .
$$

By using these coordinates it becomes clear that the number of fixed points of the Hamiltonian motion, as well as the shape of the Hamiltonian flows, are essentially determined by the parameter $\kappa_{3}$, with $K_{2}$ playing the role of a scaling parameter. Indeed, some caution is needed when interpreting the nondimensionalized coordinates. Since the coordinates are scaled by $K_{2}$, the nondimensionalized phase space will not only be scaled, but also rotated by $180 \mathrm{deg}$ compared to the normalized phase space in cases where $K_{2}<0$. Hence, the nondimensionalized polar coordinates are related to the polar coordinates in normalized phase space by,

$$
\hat{A}=\left|K_{2}\right| A, \quad \hat{\theta}= \begin{cases}\theta & \text { if } K_{2}>0 \\ (\theta+\pi) \bmod 2 \pi & \text { if } K_{2}<0 .\end{cases}
$$

A derivation of the Kobayashi Hamiltonian including arbitrary multipoles in modern notation can be found in [11].

A visual overview of the phase-space morphology as resonance is crossed is given for $\kappa_{3}=2$ and various values of $\varepsilon$ in the Hamiltonian flows of Fig. 6. The following regimes can be identified:

$\kappa_{3} \varepsilon>1 / 8$ : The only fixed point is at the origin of phase space.

$\kappa_{3} \varepsilon=1 / 8:$ Next to the fixed point at the origin, there are three more fixed points at $\hat{A}=\frac{1}{3\left|\kappa_{3}\right|}$.

$\kappa_{3}, \varepsilon>0$ : The three additional fixed points are at $\hat{\theta}=60,180$, and $300^{\circ}$.

$\kappa_{3}, \varepsilon<0$ : The three additional fixed points are at $\hat{\theta}=0,120$, and $240^{\circ}$.

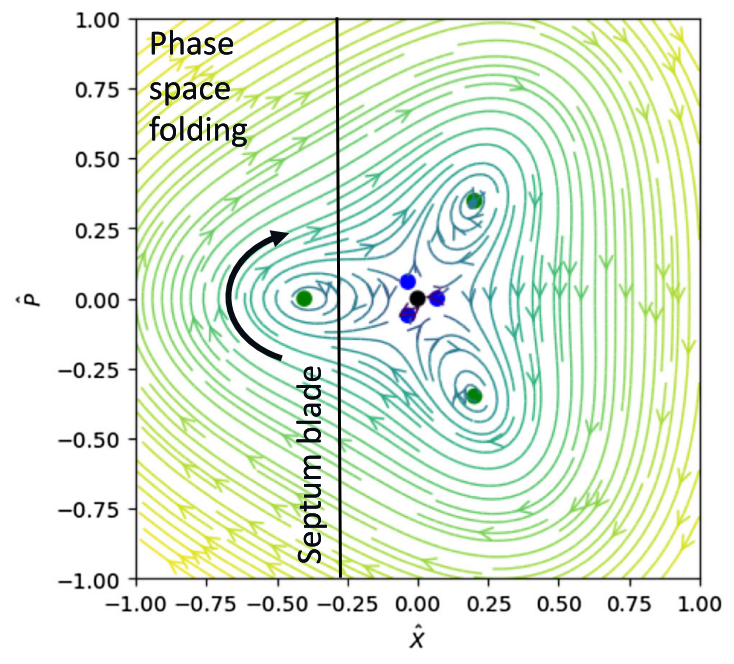

(a) $\kappa_{3} \varepsilon=-1 / 8$

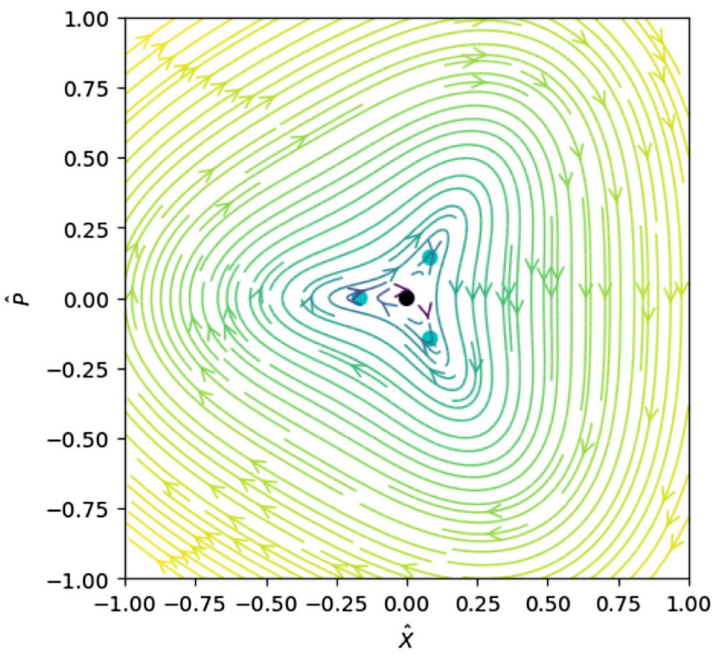

(b) $\kappa_{3} \varepsilon=1 / 8$

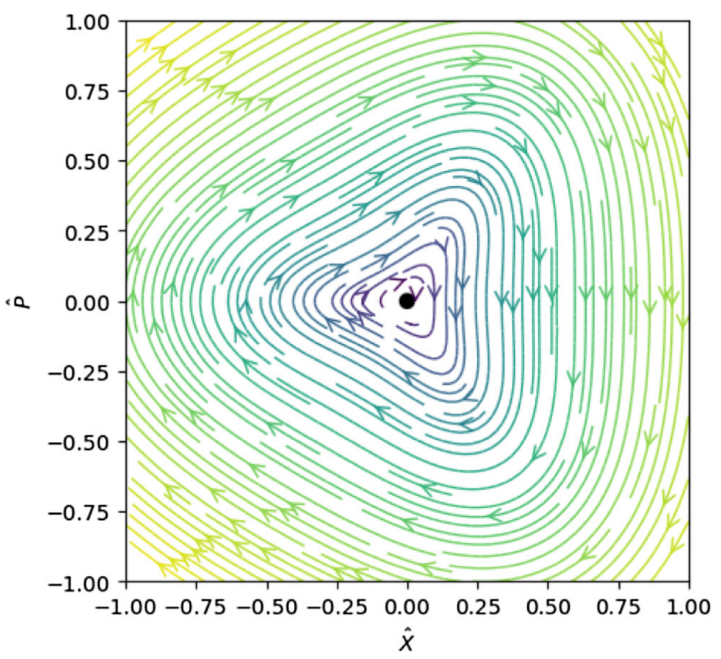

(c) $\kappa_{3} \varepsilon=1 / 4$

FIG. 6. Hamiltonian flows in nondimensionalized coordinates, for $\kappa_{3}=2$ and several values of $\varepsilon$ as the third-integer resonance is crossed. Stable fixed points (green) and unstable fixed points (blue). 
$0<\kappa_{3} \varepsilon<1 / 8$ : Next to the fixed point at the origin, there are six more fixed points at $\hat{A}=$ $\frac{1}{3\left|\kappa_{3}\right|}\left(1 \pm \sqrt{1-8 \kappa_{3} \varepsilon}\right)$.

$\kappa_{3}, \varepsilon>0$ : The six additional fixed points are at $\hat{\theta}=60,180$, and $300^{\circ}$.

$\kappa_{3}, \varepsilon<0$ : The six additional fixed points are at $\hat{\theta}=0,120$, and $240^{\circ}$.

$\varepsilon=0$ : Next to the fixed point at the origin, there are three more fixed points at $\hat{A}=\frac{2}{3\left|\kappa_{3}\right|}$.

$\kappa_{3}>0$ : The three additional fixed points are at $\hat{\theta}=60,180$, and $300^{\circ}$.

$\kappa_{3}<0$ : The three additional fixed points are at $\hat{\theta}=0,120$, and $240^{\circ}$.

$\kappa_{3} \varepsilon<0$ : Next to the fixed point at the origin, there are six more fixed points at $\hat{A}=\frac{1}{3\left|\kappa_{3}\right|}\left(\sqrt{1-8 \kappa_{3} \varepsilon} \pm 1\right)$. $\kappa_{3}>0, \varepsilon<0$ : The three additional fixed points at the smaller amplitude are at $\hat{\theta}=0^{\circ}, 120^{\circ}, 240^{\circ}$, while those at the larger amplitude are at $\hat{\theta}=60,180$, and $300^{\circ}$.

$\kappa_{3}<0, \varepsilon>0$ : The three additional fixed points at the smaller amplitude are at $\hat{\theta}=60,180$, and $300^{\circ}$, while those at the larger amplitude are at $\hat{\theta}=0,120$, and $240^{\circ}$.

The number of stable points is determined by $\kappa_{3} \varepsilon$. For nonzero $\kappa_{3}$ the motion is always bounded, regardless of the initial conditions. The motion for $\kappa_{3} \varepsilon>1 / 8$ is uninteresting with regard to extraction. For $\kappa_{3}<1 / 8$ the three fixed points at larger amplitude are stable, while the three fixed points at smaller amplitude are unstable but define a separatrix on which the motion is useful for extraction. Particles near this separatrix can grow in amplitude and then turn around the stable fixed points before decreasing in amplitude again, allowing the extraction of a beam that is folded in phase space shown schematically in Fig. 6(a).

\section{MACHINE PROTECTION ASPECTS}

The powering of octupoles during slow extraction has potentially serious machine protection implications for the delicate wire arrays in the electrostatic septum. A detailed procedure [16] was prepared and approved to verify and establish safe limits for the machine parameters applied during the tests.

The main risk was identified as accidentally trapping the beam in the machine at large amplitude with relatively strong octupole fields and collimating the beam directly onto the septum's wires. The damage limit of the $\varnothing 60 \mu \mathrm{m}$ wires in the first two septum units could be estimated from past incidents and flying wire tests carried out in the 1990s at approximately $2 \times 10^{12}$ protons. In order to mitigate the risk, a low intensity beam of $1-5 \times 10^{11}$ protons, a factor of 100 below the nominal intensity, was prepared in the CERN injector chain. An intensity on the higher end of this scale was needed in order to attain a high enough signal-to-noise ratio on the beam profile measurements taken with secondary-emission grids in LSS2.

Conversely, there was also the risk of accidentally increasing the extracted beam size at the septum and striking the cathode directly on the outside of the extraction aperture, nominally spaced at $20 \mathrm{~mm}$ from the wires. Although any risk was largely mitigated by the low intensity of the beam, a fortuitously-located LHC-type collimator (TCSM) with $1 \mathrm{~m}$ long graphite jaws, was used to safely define and restrict the machine's aperture with close to an integer number of betatron wavelengths upstream of the ZS.

\section{MAD-X STUDIES AND TRACKING RESULTS}

A simulation framework based on thin-lens tracking in MAD-X [17] was developed in order to simulate the slow extraction dynamics in the SPS under different scenarios. The framework distributes the simulations and parallelizes them over several remote machines in order to decrease the simulation time of the extraction process. The machine parameters can be varied dynamically through the extraction on a turn-by-turn basis, e.g., to simulate the behavior of the sweeping tune or a dynamic extraction bump, or held static to simulate an instance during the spill. The simulations are relatively computationally intensive because of the large number of elements in the SPS MAD-X sequence through which particles are tracked. Tracking in polymorphic maps of the SPS lattice computed using PTC [18] truncated at a given order is presently being investigated with future optimization studies in mind for the octupole extraction scheme. Recent efforts made in this direction for slow extraction studies can be found reported in $[19,20]$.

\section{A. Rotating the resonance driving term}

The amplitude detuning introduced as the octupoles are powered changes the angle at which the extracted separatrix arm strikes the septum blade. During the first beam tests it quickly became apparent that this effect needed to be compensated due to the inhibitive beam loss in LSS2, localized mainly on the TPST protection device in front of the magnetic septa. The mechanical realignment of all the extraction septa in LSS2 was ruled out as prohibitively time-consuming and impractical as the machine had to be returned to operation after the tests with only a short handover time.

Previous studies [21] showed the complexity of operating two orthogonal bumps (position and angle) superimposed on the LSS2 extraction bump to control the presentation of the separatrix to the septum.

Instead, the phase of the resonance (sextupole) driving term was rotated by using two other independent and orthogonal groups of extraction sextupoles, which remain 


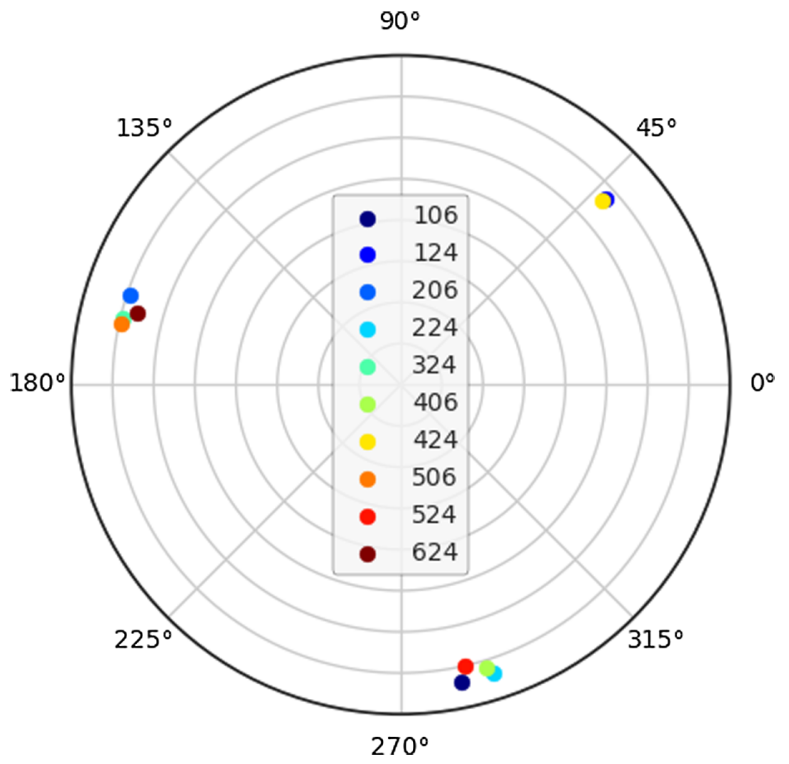

FIG. 7. Phase and amplitude of the resonance (sextupole) driving terms for each of the SPS extraction sextupoles (LSE): the naming reflects the sextant in which they are located and the quadrupole next to which they are installed, e.g., 424 is adjacent to the 24th quadrupole in sextant 4 . The four LSE sextupoles used in regular operation are grouped at a relative phase close to 290 deg.

in the SPS for historical reasons. The three groups of extraction sextupoles in the SPS can be distinguished by their relative phase advances, as computed with PTC and illustrated in Fig. 7. The reader is referred to literature [22-24] for further details on resonance driving terms and a complete description of the normalization of an effective Hamiltonian in resonance bases. The driving term angles of the four LSE sextupole magnets used in regular operation $(106,224,406$ and 524) are grouped at a relative phase of approximately $290 \mathrm{deg}$. An explanation of the naming convention is given in the caption of Fig. 7. The two other groups of sextupoles can be identified at a phase difference of 120 and $240 \mathrm{deg}$, one with four sextupoles (206, 324, 506 , and 624) and the other with two sextupoles (124 and 424). These other two groups, which are close to orthogonal, were used to change the driving term phase in a linear fashion without changing its amplitude. Changing the phase of the resonance driving term has the effect of rotating the angle of the separatrix arm presented to the septum without changing the strength of the driving term. The application of the rotation on the nominal extraction is presented in Fig. 8 with the rotation angle $\left(\Delta x_{\mathrm{rot}}^{\prime}\right)$ defined as the rotation of the separatrices in the normalized phase space plane.

\section{B. Understanding the extraction dynamics}

Tracking simulations of the extraction dynamics were used to guide the settings applied in the machine. The strength of the LOF octupoles, LSE sextupoles, LSS2 extraction bump and the sextupole rotation knob were

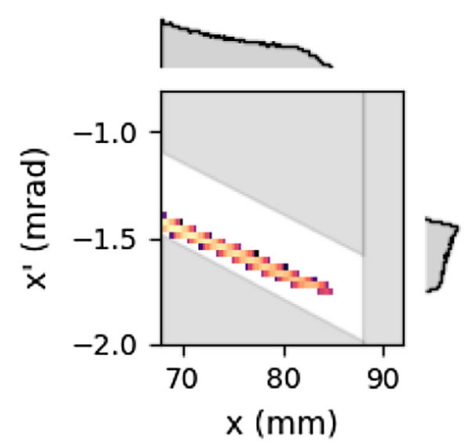

(a) $\Delta x_{\text {rot }}^{\prime}=7.6 \mathrm{deg}$

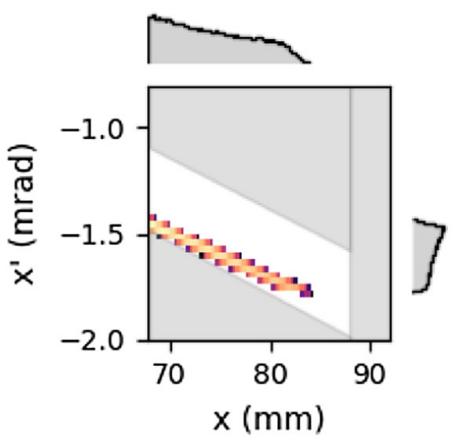

(b) $\Delta x_{\text {rot }}^{\prime}=0 \mathrm{deg}$

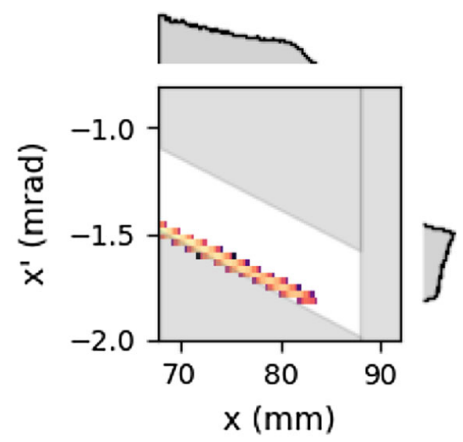

(c) $\Delta x_{\mathrm{rot}}^{\prime}=-7.6 \mathrm{deg}$

FIG. 8. Extracted beam in phase space on the high-field side upstream of the ZS simulated with different values of the resonance driving term rotation knob applied with the nominal LSE strength and extraction bump amplitude.

scanned systematically in simulation. The behavior of these main parameters are summarized qualitatively in Figs. 9-12 with high extraction sextupole strengths. The phase space is plotted together with the acceptance of the LSS2 extraction channel previously presented in Fig. 1(b). The spatial and angular distributions are also projected to illustrate the variation of the changing beam density at the $\mathrm{ZS}$. The integrated octupole gradient $\left(k_{3} L\right)$ is quoted per magnet with the powering configuration presented previously in Fig. 2 and the sextupole strength quoted in terms of the nominal value $\left(k_{2, \text { nom }}\right)$. The different parameters have a similar effect on the extracted beam distribution and are difficult to disentangle, which is discussed later in the 


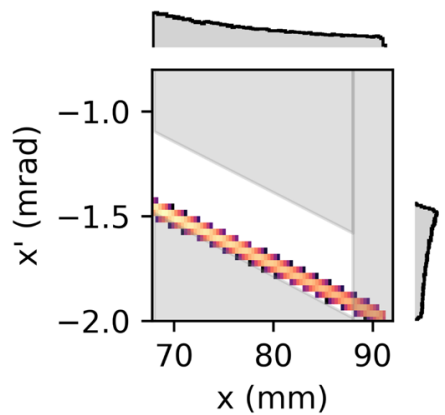

(a) $k_{3} L=0 \mathrm{~m}^{-3}$

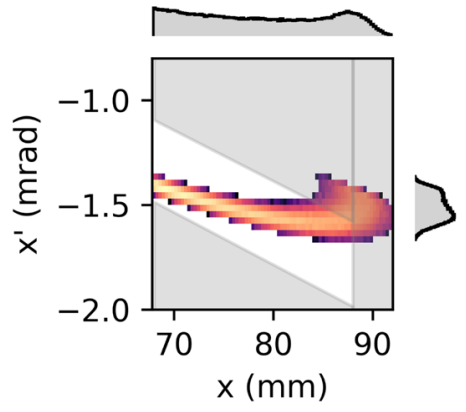

(b) $k_{3} L=-1.8 \mathrm{~m}^{-3}$

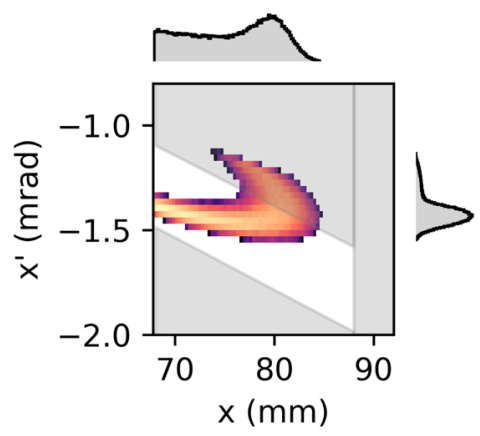

(c) $k_{3} L=-2.3 \mathrm{~m}^{-3}$

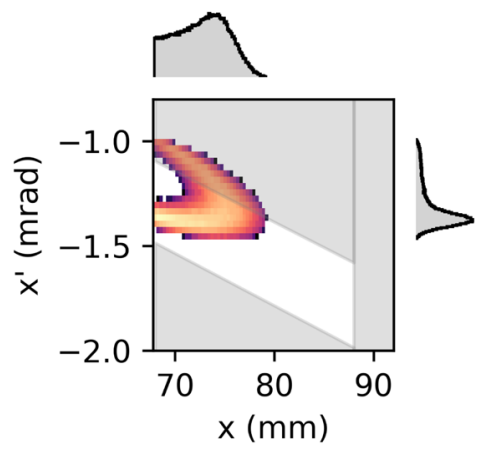

(d) $k_{3} L=-2.8 \mathrm{~m}^{-3}$

FIG. 9. Extracted beam in phase space on the high-field side upstream of the ZS simulated as a function of octupole strength with 2.1 times the nominal LSE strength, i.e., $k_{2}=2.1 k_{2 \text {,nom }}$, and nominal extraction bump amplitude. Color map is proportional to the particle density in phase space.

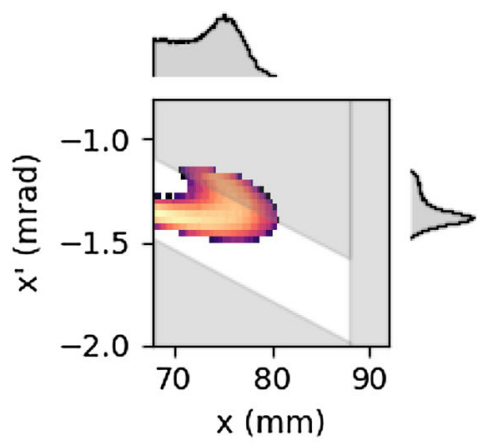

(a) $k_{2}=1.8 k_{2, \text { nom }}$

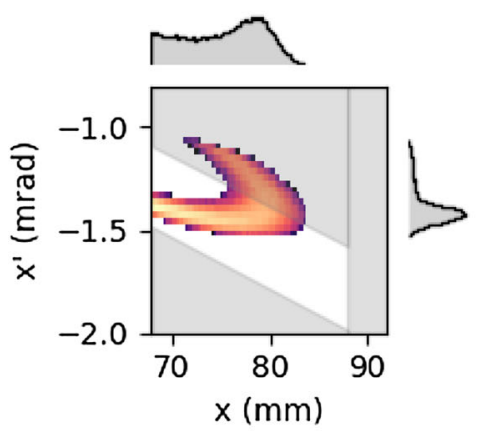

(b) $k_{2}=2.2 k_{2, \text { nom }}$

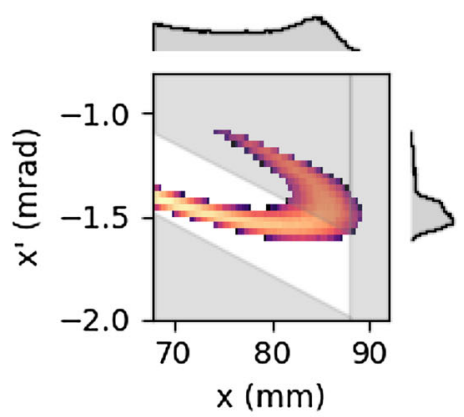

(c) $k_{2}=3.0 k_{2, \mathrm{nom}}$

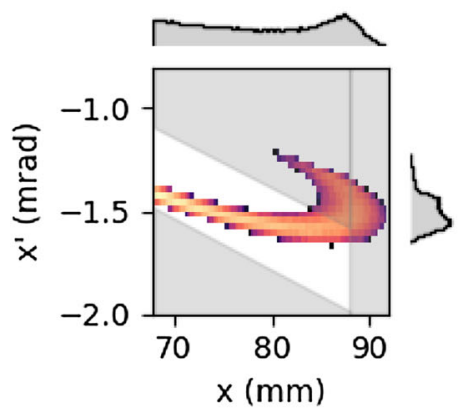

(d) $k_{2}=3.4 k_{2, \text { nom }}$

FIG. 10. Extracted beam in phase space on the high-field side upstream of the ZS simulated as a function of sextupole strength with an octupole strength of $k_{3} L=-2.45 \mathrm{~m}^{-3}$ and nominal extraction bump amplitude. 
context of the measurements. Most evident is the significant growth of the extracted beam emittance compared to the extraction channel acceptance.

\section{LOF circuit}

The separatrix can be bent downward in phase space using positive LOF strengths, or upward with negative values. Increasing the strength of the LOF circuit has the effect of stabilizing and folding the outward streaming separatrix, which is shown in Fig. 9 for an LSE strength 2.1 times higher than nominal, i.e., $k_{2}=2.1 k_{2, \text { nom }}$. The relative strength of the LSE and LOF circuits have important consequences for the beam density at the ZS. This particular example highlights the machine protection concerns for the ZS with (i) relatively low LOF strengths (small $\kappa_{3}$ ) allowing particles to be extracted directly onto the cathode (at $x>88 \mathrm{~mm}$ ) and (ii) relatively high LOF strengths (large $\kappa_{3}$ ) trapping particles close to and collimating particles directly onto the wire-array of the ZS (at $x_{\mathrm{ZS}}=68 \mathrm{~mm}$ ).

\section{LSE circuits}

The relative strength of the extraction sextupoles has the inverse effect of the octupoles, fighting the stabilization introduced by the LOF circuit, as shown in Fig. 10 for an LOF strength of $k_{3} L=-2.45 \mathrm{~m}^{-3}$ and the nominal extraction bump amplitude of $x_{\text {bump,nom }}=44.3 \mathrm{~mm}$.

\section{LSS2 extraction bump amplitude}

The extraction bump is a sensitive parameter that defines the amplitude at which particles can sample the field in the octupoles in the last turns before jumping the extraction septum. The length of the folded separatrix arm shrinks as the phase space amplitude growth $(\Delta A)$ of on-resonance particles in the last 3 turns is reduced when the beam is bumped closer to the ZS. In the absence of octupoles, the spiral step is described by the following expression,

$$
\Delta A \propto \frac{3}{4}\left|K_{2}\right| A_{\mathrm{ZS}}^{2}
$$

where the phase space amplitude between the beam and the septum blade of the ZS is given by $A_{\mathrm{ZS}}^{2}=\left(x_{\mathrm{ZS}}-x_{\text {bump }}\right)^{2}+$ $\left(x_{\mathrm{zS}}^{\prime}-x_{\text {bump }}^{\prime}\right)^{2}$. Figure 11 illustrates this behavior in the presence of strong LSE and LOF strengths.

\section{Resonance driving term rotation}

Rotation of the resonance driving term helps align the beam with the septum blade. The knob also has an impact on the folding by adjusting the amplitude growth in the final turns by adjusting the magnitude of $A_{\mathrm{ZS}}$ described by Eq. (8). Figure 12 shows that the rotation plays a similar role to the extraction bump and, as a result, the length of the folded separatrix arm varies as a function of the rotation.

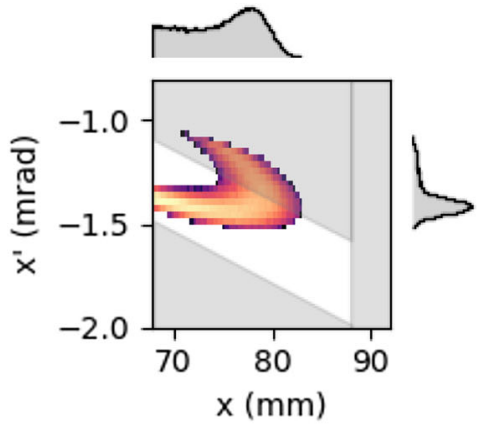

(a) $x_{\text {bump }}=44.3 \mathrm{~mm}$

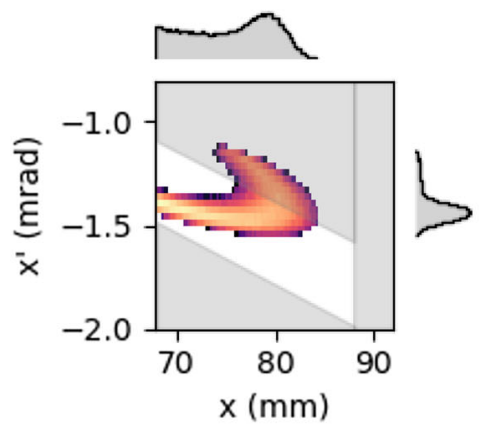

(b) $x_{\text {bump }}=45.8 \mathrm{~mm}$

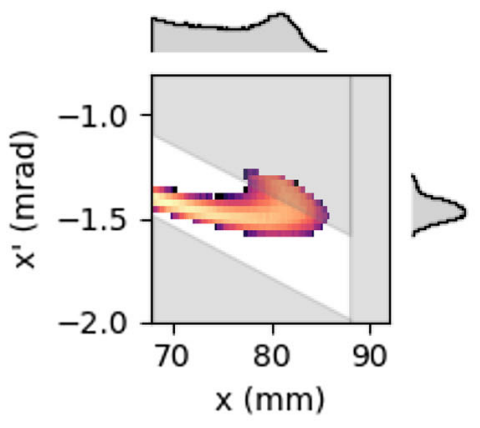

(c) $x_{\text {bump }}=47.3 \mathrm{~mm}$

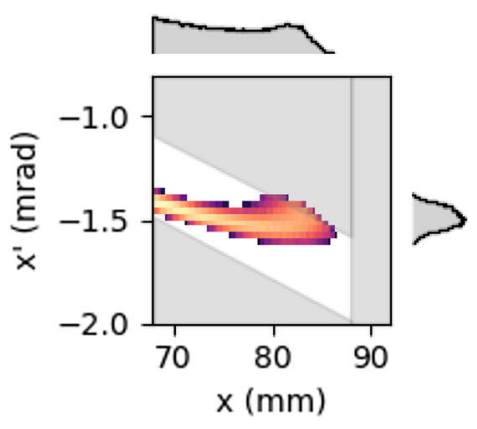

(d) $x_{\text {bump }}=48.7 \mathrm{~mm}$

FIG. 11. Extracted beam in phase space on the high-field side upstream of the ZS simulated as a function of extraction bump amplitude strength with 2.1 times the nominal LSE strength, i.e., $k_{2}=2.1 k_{2, \text { nom }}$, and octupole strength $k_{3} L=-2.45 \mathrm{~m}^{-3}$. 


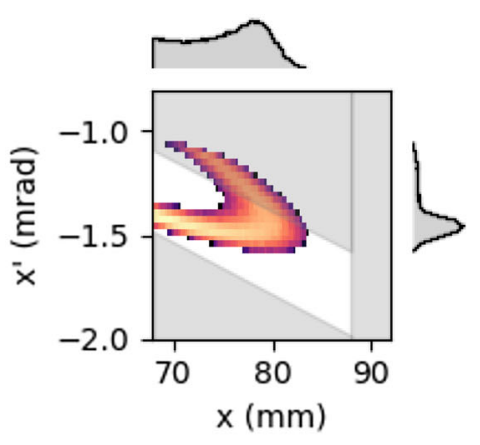

(a) $\Delta x_{\text {rot }}^{\prime}=-4.6 \mathrm{deg}$

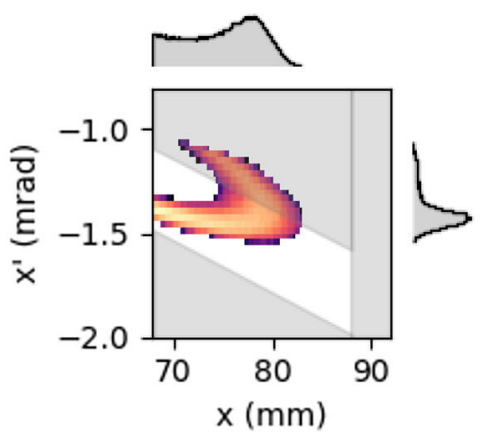

(b) $\Delta x_{\mathrm{rot}}^{\prime}=-1.5 \mathrm{deg}$

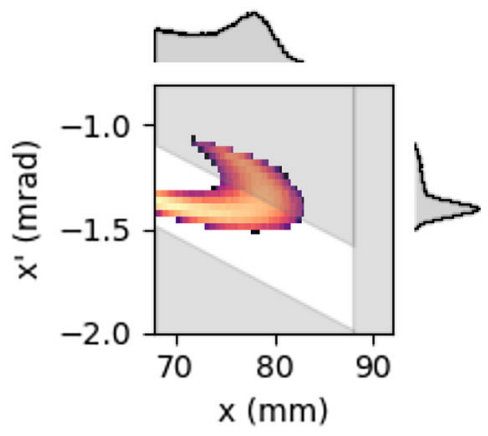

(c) $\Delta x_{\mathrm{rot}}^{\prime}=1.5 \mathrm{deg}$

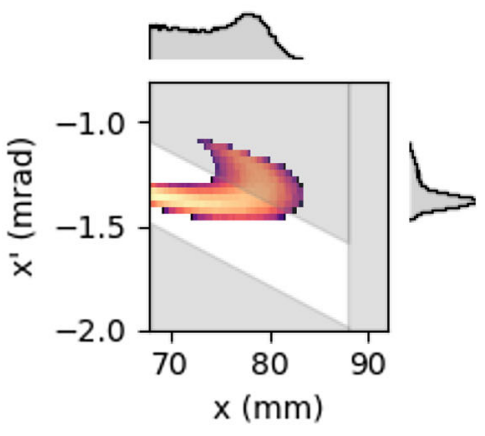

(d) $\Delta x_{\text {rot }}^{\prime}=4.6 \mathrm{deg}$

FIG. 12. Extracted beam in phase space on the high-field side upstream of the $\mathrm{ZS}$ simulated as a function of resonance driving term rotation knob $\left(\Delta x_{\text {rot }}^{\prime}\right)$ with 2.1 times the nominal LSE strength, i.e., $k_{2}=2.1 k_{2, \text { nom }}$, and octupole strength $k_{3} L=-2.45 \mathrm{~m}^{-3}$.

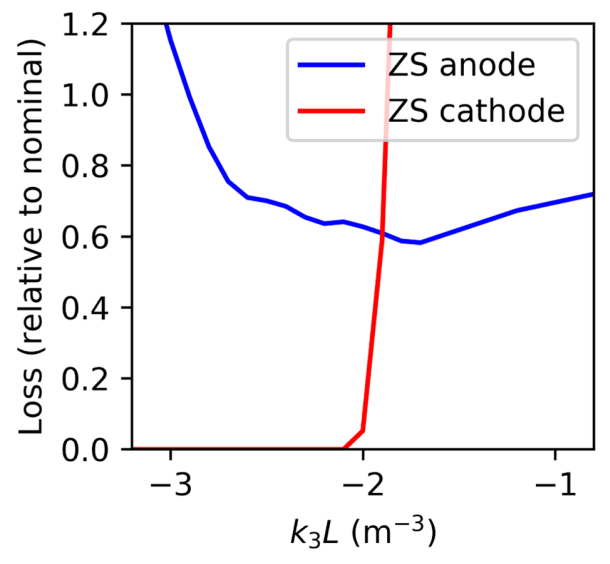

(a) LOF $\operatorname{scan}\left(k_{2} L=2.1 k_{2, \text { nom }}\right)$

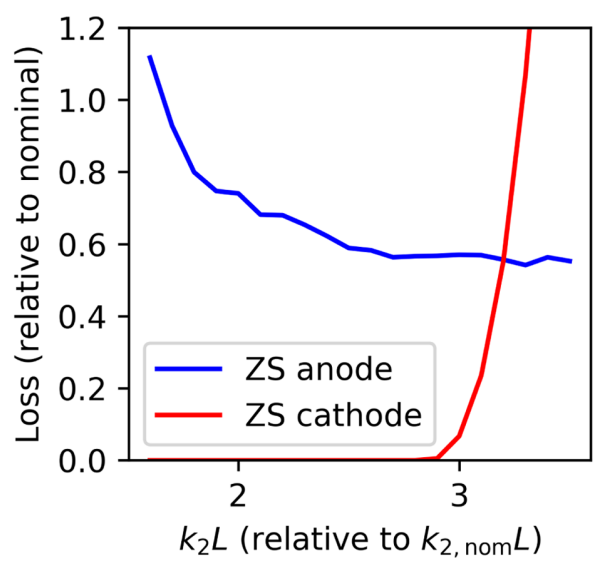

(b) LSE scan $\left(k_{3} L=-2.45 \mathrm{~m}^{-3}\right)$

FIG. 13. Simulated beam loss at the ZS as a function of the LSE and LOF strength applied.

This behavior complicates the operational setup of the extraction.

\section{Loss reduction potential}

The effect of the changing beam distribution on the extraction efficiency is summarized in Fig. 13 as a function of the sextupole and octupole strengths presented in the parametric simulation studies. The relative beam loss is computed by comparing the number of particle hits on the geometry of the extraction septum modeled as a black absorber. The effective thickness of the wire array was taken as $500 \mu \mathrm{m}$, which is much larger than the diameter of the individual wires strung on the ZS anode but consistent with recent independent measurements reported in $[19,25,26]$. The angular spread of the beam impacting the septum blade is increased in the presence of strong multipoles, as parametrized in Fig. 14. However, with such a large effective septum blade thickness the effect of the angular spread on the loss at the septum is negligible. The simulation results indicate that a loss reduction of up to approximately $40 \%$ is possible at the extraction septum. 


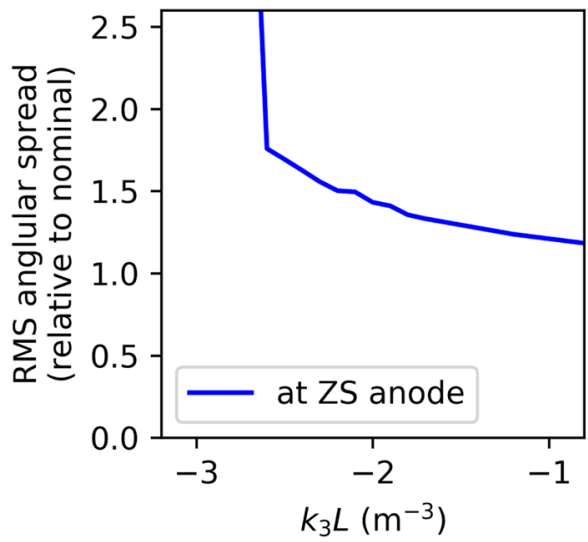

(a) LOF scan $\left(k_{2} L=2.1 k_{2, \text { nom }}\right)$

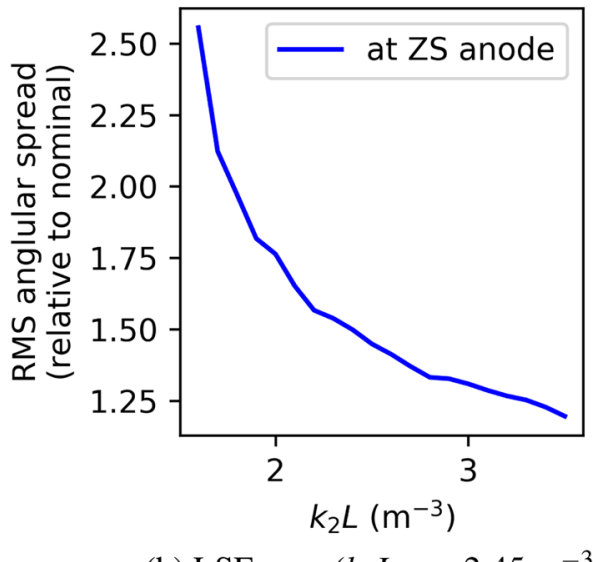

(b) LSE scan $\left(k_{3} L=-2.45 \mathrm{~m}^{-3}\right)$

FIG. 14. Simulated angular spread impacting the ZS anode wire-array as a function of the LSE and LOF strength applied.

The increased angular spread of the separatrix arm will play a significant role in the extraction efficiency when octupoles are applied in combination with the crystal shadowing technique, where the angular acceptance of the crystal is limited, and if the effective thickness of the septum blade is reduced. The dependence of the channeling efficiency as a function of the angular spread of the beam is presented in [19]. The increased angular spread of approximately $25 \%$ is expected to reduce the channeling efficiency by $20 \%$ at $400 \mathrm{GeV} / c$ in the SPS.

As mentioned above, systematic simulation studies are presently underway using PTC to understand the full loss reduction potential of octupoles at the SPS as a function of effective septum blade thickness and multipole strengths.

\section{Error studies}

The closed-orbit at flat-top is measured and corrected once per year during the recommissioning period by voluntarily displacing selected quadrupoles. This approach is taken because the SPS has no orbit corrector magnets effective at $400 \mathrm{GeV} / c$. The procedure is always a compromise because the closed-orbit is different for the different optics in the cycles played, e.g., $450 \mathrm{GeV} / c$ LHCtype cycles compared to the $400 \mathrm{GeV} / c$ fixed-target cycle. As a result, a relatively large nonzero root-mean-square (rms) closed-orbit of $\sim 2 \mathrm{~mm}$ measured on the beam position monitor (BPM) system is not unusual.

An extensive simulation effort was carried out before any beam tests to understand the behavior of feed-down in the octupoles on the resonant dynamics. An error study was performed using Gaussian alignment errors on all main quadrupoles with an rms of $150 \mu \mathrm{m}$ in order to create random error seeds with closed-orbits comparable to those measured in the SPS. It was observed that: (i) The orbit variation driven by ramping the octupole and sextupole strength is not significant. A maximum change of $70 \mu \mathrm{m}$ in the $\mathrm{rms}$ of the closed-orbit is expected for worst-case error seeds and strongest octupole fields; (ii) The nonzero closed-orbit offset in the octupoles can change the sextupole driving term strength by up to a few percent; (iii) Feed-down from the octupoles may change the angle of the sextupole driving term, but the change is anyway small compared to the uncertainty coming from the nonzero closed-orbit in the sextupoles.

Likewise, an error study was performed investigating the effect of $\beta$-beating on the resonance driving term in the presence of a non-zero closed-orbit. For the expected level of $\beta$-beating in the SPS the effect on the sextupole driving term can be up to $~ 5 \%$ in strength and 20 deg in angle. All of these effects were expected to have minimal effect on the extraction and without risk to the machine as the octupoles are powered. More details of the error study can be found in [11].

\section{SLOW EXTRACTION TESTS}

The beam tests were performed over two mornings and totalled no more than $8 \mathrm{~h}$ due to the reduced availability of the injector chain on those days. They were carried out with an intensity of approximately $5 \times 10^{11}$ protons, extracted with COSE on a spill with a flat-top duration of $4.8 \mathrm{~s}$.

The tests were split into two parts. In order to safely check the effective strength of the multipoles in the machine and their interplay, a first step was carried out with the LSS2 extraction bump off and the beam safely slow extracted onto the TCSM collimator located in LSS5 of the SPS. The second part proceeded with extraction through LSS2 onto a beam dump located at the upstream end of the transfer line headed toward the North Area. Therefore, the transport and splitting of the beam in the transfer line was not studied. The main objectives of the procedure were to safely: (1) Demonstrate and understand the possibility to resonantly drive the beam to large amplitude with sextupoles and trap it with octupoles, checking the strength of the multipole terms in the machine with those implemented in simulation; (2) Demonstrate that the changing angle of the extraction separatrix at the septum, induced by the amplitude detuning of the 


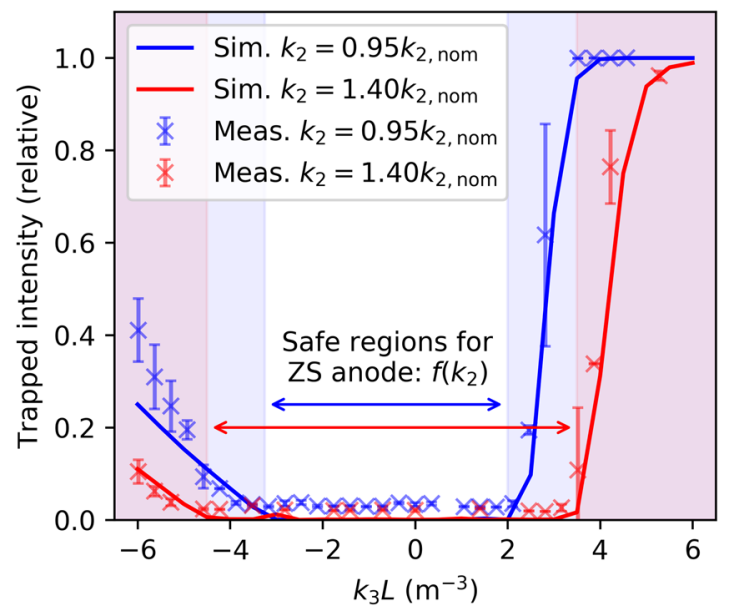

FIG. 15. Trapped beam intensity circulating at the end of the flat-top, and subsequently dumped, as a function of the LOF strength, with the TCSM aligned at the equivalent aperture of the $\mathrm{ZS}$ wire-array. The shaded regions identify the parameter space to be avoided to protect the wire-arrays of the ZS from the risk of trapping at large amplitude.

octupoles, can be compensated by adjusting the phase of the sextupole resonance driving term; (3) Demonstrate clear changes in the extracted beam profile and extraction losses in LSS2 with varying octupole and sextupole strengths; (4) Demonstrate prompt extraction beam loss reduction at the electrostatic extraction septum with carefully chosen sextupole and octupole strengths.

\section{A. Verification of multipole strengths}

The machine's aperture bottleneck was transferred from the extraction septum to the outer jaw of the TCSM collimator. This was achieved with a beam-based alignment whilst nominally slow extracting to the transfer line dump. The outer TCSM jaw was inserted into the beam's separatrix arm on its final turn in the machine until the loss signal at the extraction septum in LSS2 dropped sharply, along with the extracted beam intensity. The evolution of the trapping process was observed on the circulating beam intensity measured throughout the spill and beam profile measurements of the circulating beam.

With the TCSM aligned to the equivalent aperture of the ZS wire-array, the extraction bump in LSS2 was turned off and the beam slow extracted onto the TCSM. The LOF circuit could then be powered safely without any risk to the ZS. The behavior of the beam loss measured at the TCSM and the beam intensity trapped at the end of the flat-top was used to validate the multipole strengths applied by comparing the onset of beam trapping with simulation. The trapped intensity was preferred to the beam loss signal because the changing impact position and angle of the beam on the TCSM complicated the measured dose given to the downstream beam loss monitor (BLM). The comparison of the trapping phenomenon to simulation is shown in Fig. 15 for

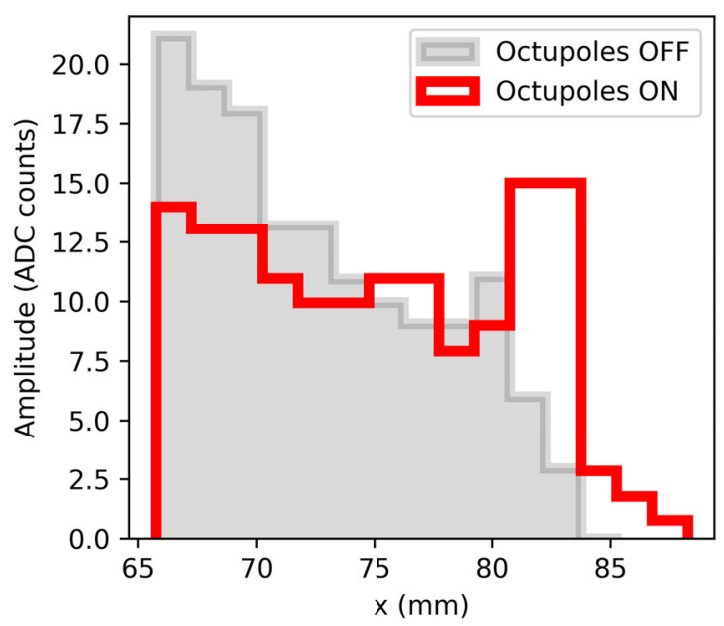

(a) Horizontal profile measurements of the extracted beam at BSGH.216, demonstrating an intensity reduction of $35 \%$ at the estimated position of the adjacent electrostatic septum blade $\left(x_{\mathrm{ZS}}=68 \mathrm{~mm}\right)$.

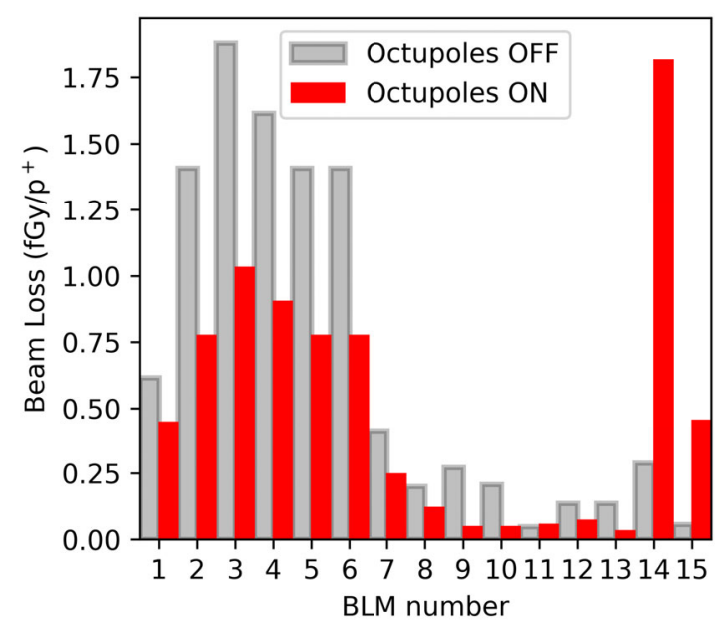

(b) Beam loss (normalised to extracted intensity) along LSS2, demonstrating a loss reduction of $42 \%$ at the first 5 BLM's next to the ZS. See Fig. 17 for the mapping of BLM location and position.

FIG. 16. Extraction measurements showing the beam distribution and beam loss with strong multipole fields powered $\left(k_{2}=2.1 k_{2, \text { nom }}\right.$ and $\left.k_{3} L=-2.45 \mathrm{~m}^{-3}\right)$.

different sextupole strengths. At weak LOF settings, the beam is slow-extracted onto the TCSM, as expected. As the LOF strength is ramped, either positively or negatively, the separatrix arms bend around the stable islands formed at large amplitude, eventually trapping the beam in the machine. Within the tolerances on the measured beam size and position of the TCSM, the onset of trapping is well predicted by simulation for both positive and negative values, which allowed the extraction tests to continue with confidence. The same exercise was repeated for stronger LSE strengths and demonstrated that higher LOF strengths are indeed needed to trap the beam. The exercise allowed safe limits to be defined for the relative LSE and LOF strengths to protect the ZS anode, as shown in Fig. 15. 


\section{B. Rotating the resonance driving term}

Without the LOF circuit powered, the rotation of the driving term was validated by comparing the shape and center-of-mass of the extracted beam distribution on two beam profile monitors located immediately upstream of the ZS and approximately $90 \mathrm{deg}$ phase advance further downstream inside the MSE septum. The beam loss measured using the BLM system installed along LSS2 showed good agreement with a movement of the downstream end of the girder supporting the ZS, which is an analogous way of changing the relative angular alignment of the beam and ZS.

The ability to rotate the angle at which the extracted separatrix is presented to the septum also has an important operational relevance for the septum alignment independent of the discussion of extraction with octupoles in this paper. Typically, the electrostatic septa have to be realigned during the operational year due to a slowly drifting closedorbit, which is a time-consuming mechanical procedure. In the future, the rotation knob will be implemented into the alignment procedure to speed up and improve its reproducibility.

The extracted beam position and size measured in the presence of octupole fields showed good agreement with simulation. The ability to compensate for the changing angle allowed the machine tests to progress without realigning the extraction channel. Further details of these aspects can be found detailed in [27].

\section{Extraction measurements with octupoles}

Although it would have been preferable to systematically scan and optimize all multipole parameters, the limited time meant that only a few combinations of octupole and sextupole strengths, which performed well in simulation, were tested. For each setting, a scan of the rotation knob was performed to realign the beam with the ZS by minimizing the sum of the loss signal measured on the BLM monitors next to the ZS.

Unfortunately, it became clear toward the end of the tests that the TCSM, which was aligned to protect the ZS cathode from direct beam impact, was too close to the edge of the beam and was perturbing the measurements of the horizontal profile distribution when strong multipole strengths were applied. Once the TCSM was retracted, the phase space folding was clearly demonstrated on the measured beam profile distribution, as shown in Fig. 16(a) for the best loss reduction settings. The modified beam density distribution at the entrance to the extraction septum shows a clear reduction of approximately $35 \%$ in the beam intensity close to the estimated ZS wire location and an increased density at large amplitude. The settings applied with the TCSM retracted for this case were $k_{2}=2.1 k_{2, \text { nom }}$ and $k_{3} L=-2.45 \mathrm{~m}^{-3}$. A corresponding loss reduction of $42 \%$ was measured on the sum of the 5 BLMs next to the extraction septum, as shown by the beam loss profile measured along LSS2 in Fig. 16(b). In this case, the beam loss downstream in LSS2 increased because of the large emittance of the extracted beam. As the beam was rotated using the driving term rotation knob, the losses at the electrostatic septum decreased but the loss signal on BLMs located close to the enlarged aperture quadrupole (QDA.219) increased. The loss increase was most likely caused by the beam scraping on the outside of the downstream end of the MSE. The trajectory of the simulated distributions through the LSS2 extraction channel is shown in Fig. 17 along with the location and coverage of the BLM and BSGH instrumentation devices. Due to the limited time available it was not possible to attempt to reduce the beam

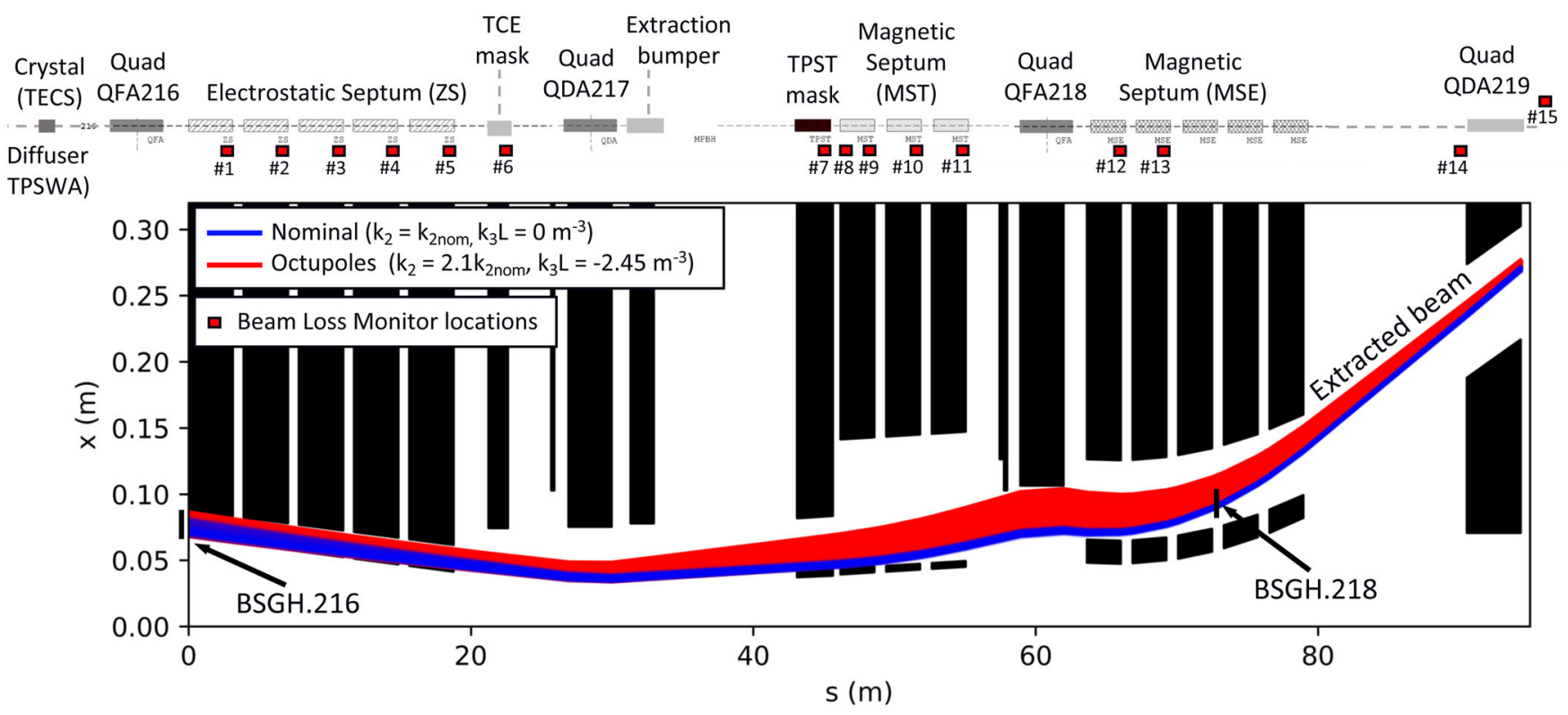

FIG. 17. Extracted beam trajectories along LSS2 with the multipoles powered $\left(k_{2}=2.1 k_{2, \text { nom }}\right.$ and $\left.k_{3} L=-2.45 \mathrm{~m}^{-3}\right)$. 


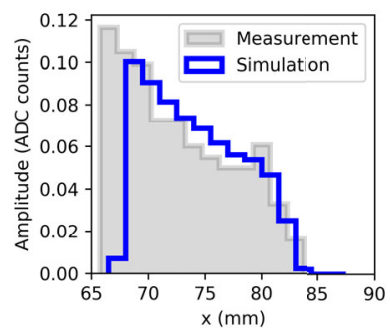

(a) $k_{2}=k_{2, \text { nom }}, k_{3} L=0 \mathrm{~m}^{-3}$

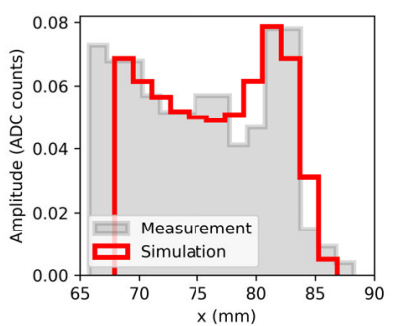

(b) $k_{2}=2.1 k_{2, \text { nom }}, k_{3} L=-2.45 \mathrm{~m}^{-3}$
FIG. 18. Horizontal beam profile at BSGH.216 upstream ZS.

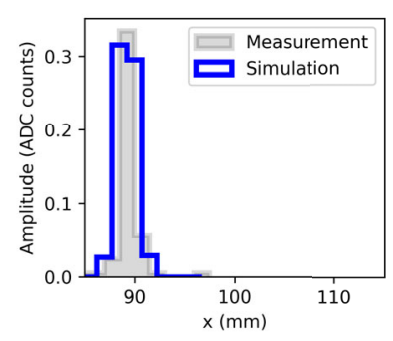

(a) $k_{2}=k_{2, \text { nom }}, k_{3} L=0 \mathrm{~m}^{-3}$

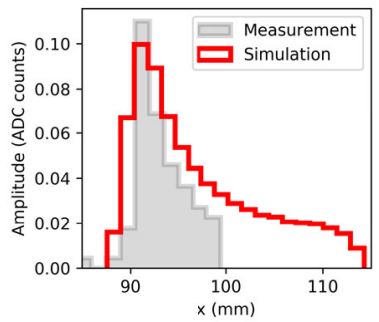

(b) $k_{2}=2.1 k_{2, \text { nom }}, k_{3} L=-2.45 \mathrm{~m}^{-3}$
FIG. 19. Horizontal beam profile at BSGH.218 inside MSE.

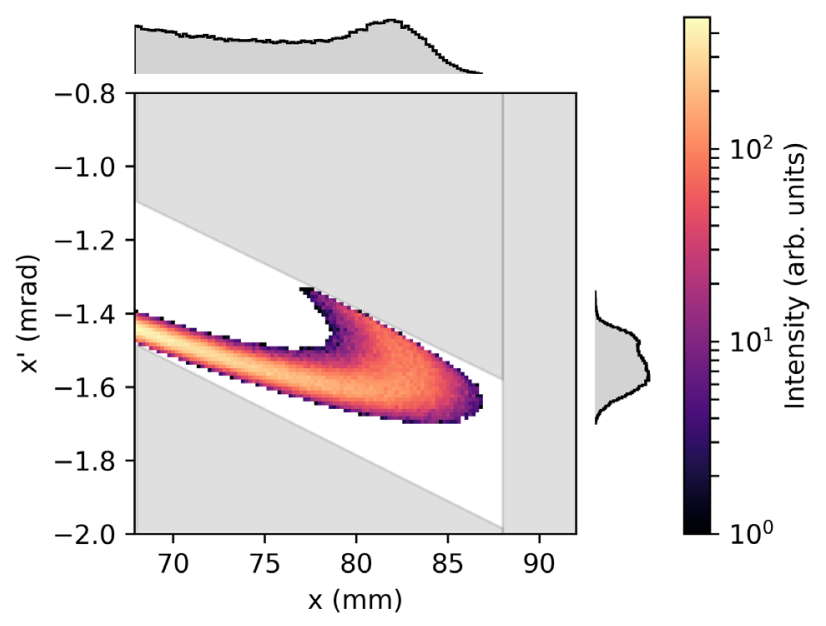

FIG. 20. Extracted beam in phase space on the high-field side upstream of the ZS simulated fitted to the measured beam profile distribution with the multipoles powered $\left(k_{2}=2.1 k_{2, \text { nom }}\right.$ and $k_{3} L=-2.45 \mathrm{~m}^{-3}$. The acceptance limit of the LSS2 extraction channel is shaded grey.

loss observed downstream by steering or realigning the extraction channel.

The applied multipole strengths were implemented in simulation along with a rotation of the resonance driving term to align the beam and septum, before being fitted to the beam profile measured at BSGH.216 using the LSS2 extraction bump amplitude as the free parameter. A comparison between measurement and simulation is shown in Figs. 18 and 19. The large beam size at
BSGH.218 highlights the increased divergence of the extracted beam, which is not evident $90 \mathrm{deg}$ upstream at the ZS. The limited coverage of the grid of BSGH.218 $( \pm 10 \mathrm{~mm})$ is exposed by the missing data at amplitudes larger than about $100 \mathrm{~mm}$. The simulated phase space distribution at the upstream end of the ZS in Fig. 20 shows how the acceptance of the LSS2 extraction channel is filled leaving very little, if any, margin for misalignment.

\section{Discussion}

The measured loss reduction was larger than expected from the modification to the beam profile distribution observed on BSGH.216 upstream the ZS. However, the density reduction at the septum wire-array is difficult to infer due to the poor resolution (grid width of $1.5 \mathrm{~mm}$ ), noise at the low intensities used and the uncertainty on the relative position between the grid and septum blade. The increased loss reduction could also be explained by the fact that no alignment, either mechanical or with the rotation knob, was carried out for the nominal case without the LOF circuit powered. It had been several months since the last beam-based alignment of the $\mathrm{ZS}$.

The simulations including strong multipoles required the extraction bump amplitude to be scaled-up by a few percent to achieve good agreement with measurements. This discrepancy amounts to a closed-orbit perturbation of approximately $2 \mathrm{~mm}$ at the ZS induced by feed-down in the strong multipoles, which is much larger than expected. As pointed out previously, each of the available degrees of freedom play a similar role in the distribution of the beam profile measured at the ZS. It is more likely that the discrepancy is due to an error in the effective multipole strength arising from the nonzero closed-orbit or magnet calibration and transfer function errors. More information is required to better constrain the simulations. The changing closed-orbit position in LSS2 was not measured during the beam tests and, although the multipole strengths were validated (see Fig. 15) as best as possible, the measurement was indirect and includes significant errors from the beam size and alignment tolerances at the TCSM. In the future, closed-orbit and optics measurements will be needed to better constrain the simulation model.

The impact of the strong multipoles on the vertical emittance was not measured as a function of time during the flat-top and will be the subject of future investigations, however the emittance growth is expected to be negligible.

\section{CONCLUSION}

First tests applying octupoles during slow extraction to manipulate the phase space density of the beam at the electrostatic extraction septum have successfully demonstrated the feasibility of the principle at the CERN SPS and validated the simulation tools used to understand the extraction dynamics. Although the location of the octupoles 
in regions of high dispersion is not ideal, the beam loss at the extraction septum was reduced by over $40 \%$ with carefully chosen multipole strengths. The increased beam emittance was problematic in the limited acceptance of the extraction channel and the beam loss increased as a result in the downstream end of LSS2 as the beam left the synchrotron. Although the computed acceptance indicates that the extraction of the larger emittance is possible, no attempt could be made to realign the extraction channel with the beam due to the limited time available.

\section{OUTLOOK}

To implement the octupole scheme in operation, further study and optimization of the machine, e.g., steering and alignment of extraction septa in LSS2 etc., will be needed after the SPS restarts from Long Shutdown 2 in 2021 to ensure that the increased horizontal transverse emittance can be transported out of LSS2, split in TT20 and transported to the production targets. In addition, the machine protection aspects of an operational implementation will need careful consideration. Studies are on-going to understand the maximum loss reduction potential in the SPS with all constraints implemented, however, the studies presented in this paper indicate that a loss reduction of greater than $40 \%$ is unlikely when the maximum sextupole strength, which amounts to $k_{2}=3 k_{2 \text { nom }}$, is considered.

The tests have highlighted the challenges of decoupling the effects of the different degrees of freedom when setting up the extraction and the need for improved beam instrumentation in LSS2, and in the SPS in general. In particular, the ability to accurately measure the nonlinear optics and validate the applied strengths at flat-top will be important to constrain the simulation model of the extraction. Studies are already underway to apply advanced numerical optimization algorithms to the operational setup of the extraction and to maximize the loss reduction with the available parameters and constraints.

Slow extraction with octupoles was also tested with success in combination with a thin, bent crystal shadowing the wires of the ZS to achieve a combined loss reduction factor of $\sim 3$. More tests are needed to accurately measure the impact that the increased angular spread from the application of octupoles has on the effectiveness of the crystal shadowing technique in comparison to simulation. Further details can be found reported elsewhere [2]. This important result demonstrates that different loss reduction techniques can be combined in the quest for the factor of 4-5 reduction in the specific extraction loss needed to welcome the proposed BDF at CERN.

\section{ACKNOWLEDGMENTS}

The authors thank the CERN PS Booster, PS, and SPS operations teams for their efforts in making the tests possible, especially on the busy days where multiple and changing requests had to be coordinated at short notice. The same gratitude is extended to the Injector Controls Architecture (INCA) support team who were always available to adjust software limits and to the rf teams in the different machines who set-up the low intensity beam that made the safe tests possible. We appreciate the support of the Accelerator and Beam Physics group for giving us the opportunity to use their collimation equipment and the Beam Instrumentation group for ensuring smooth data acquisitions and following-up issues throughout the measurement campaign. We also thank Hannes Bartosik, Giovanni Rumolo, and Karel Cornelis for their valuable advice and careful scheduling of the machine development time.

[1] M. A. Fraser et al., SPS slow extraction losses and activation: Challenges and possibilities for improvement, in Proc. of IPAC'17, Copenhagen, Denmark (JACoW, Geneva, Switzerland, 2017), paper MOPIK045, pp. 611614.

[2] M. A. Fraser et al., SPS slow extraction losses and activation: Update on recent improvements, in Proc. of IPAC'19, Melbourne, Australia, 2019 (JACoW Publishing, Geneva, Switzerland, 2019), paper WEPMP031, pp. 23912394.

[3] A. Golutvin et al., A facility to search for hidden particles (SHiP) at the CERN SPS, CERN Report No. CERNSPSC-2015-016 (SPSC-P-350), 2015.

[4] C. C. Ahdida, M. Calviani, B. Goddard, R. Jacobsson, and M. Lamont, SPS beam dump facility comprehensive design study, CERN Report No. CERN-PBC-REPORT2018-001, 2019.

[5] K. A. Brown, V. Schoefer, and M. Tomizawa, Improving extraction efficiency of the third integer resonant extraction using higher order multipoles, BNL, Technical Report No. CA/AP/584, 2017.

[6] N. N. Alexeev, S. L. Bereznitsky, and A. E. Bolshakov, Perturbation of relevant resonance for slow extraction efficiency increase, in Proceedings of the Particle Accelerator Conference, Vancouver, BC, Canada, 1997 (IEEE, New York, 1997), pp. 261-263.

[7] V. Nagaslaev, K. A. Brown, and M Tomizawa, Third integer resonance extraction with presence of higher multipoles, Phys. Rev. Accel. Beams 22, 043501 (2019).

[8] L. S. Stoel et al., Phase space folding studies for beam loss reduction during resonant slow extraction at the CERN SPS, in Proc. of IPAC'17, Copenhagen, Denmark (JACoW, Geneva, Switzerland, 2017), paper MOPIK046, pp. 615-617.

[9] L.S. Stoel et al., Investigating beam loss reduction with octupoles during slow extraction in the CERN SPS, in Proc. of IPAC'18, Vancouver, BC, Canada (JACoW Publishing, Geneva, Switzerland, 2018), paper TUPAF051, pp. 822825.

[10] L. S. Stoel et al., Slow extraction loss reduction with octupoles at the CERN SPS, in Proc. of IPAC'19, Melbourne, Australia (JACoW Publishing, Geneva, Switzerland, 2019), paper WEPMP033, pp. 2399-2402. 
[11] L. S. Stoel, Ph.D. thesis, Low-loss resonant extraction from hadron synchrotrons in the TeV energy scale, T.U. Wien (to be published).

[12] H. Bartosik et al., Reconfiguration of SPS Landau octupole circuits to minimise second order chromaticity, in Proc. of IPAC'19, Melbourne, Australia, 9-24 May 2019, paper MOPGW072, pp. 262-265.

[13] V. Kain, F. M. Velotti, M. A. Fraser, B. Goddard, J. Prieto, L. S. Stoel, and M. Pari, Resonant slow extraction with constant optics for improved separatrix control at the extraction septum, Phys. Rev. Accel. Beams 22, 101001 (2019).

[14] L. Badano et al., Proton-Ion Medical Machine Study (PIMMS) Part 1, CERN-TERA Foundation-MedAustron Oncology-2000 Collaboration Technical Report No. CERNPS-99-010-DI, 1999, https://cds.cern.ch/record/385378.

[15] Y. Kobayashi and H. Takahashi, Improvement of the emittance in the resonant beam ejection, in Proc. of the 6th Int. Conf. on High-Energy Accelerators (Cambridge, Massachusetts, 1967), pp. 347-351.

[16] M. A. Fraser, L. S. Stoel, and F. M. Velotti, Injector MD Procedure-SPS Separatrix Folding with Octupoles during Resonant Slow Extraction, EDMS No. 2014948 (CERN, Geneva, Switzerland, 2018).

[17] Methodical Accelerator Design. https://madx.web.cern.ch.

[18] E. Forest, E. McIntosh, and F. Schmidt, Introduction to the polymorphic tracking code: Fibre bundles, polymorphic Taylor types and Exact tracking, CERN Technical Report No. CERN-SL-2002-044-AP, 2002.

[19] F. M. Velotti et al., Septum shadowing by means of a bent crystal to reduce slow extraction beam loss, Phys. Rev. Accel. Beams 22, 093502 (2019).
[20] F. M. Velotti, Speeding up numerical simulations with PTC maps at arbitrary order, in the Slow Extraction Workshop 2019, FNAL, Chicago, USA, 2019, https://indico.fnal.gov/ event/20260/session/11/contribution/26.

[21] L.S. Stoel et al., Progress toward a dynamic extraction bump for slow extraction in the CERN SPS, in Proc. of IPAC'18, Vancouver, BC, Canada (JACoW Publishing, Geneva, Switzerland, 2018), paper TUPAF055, pp. 822825.

[22] G. Guignard, The general theory of all sum and difference resonances in a three-dimensional magnetic field in a synchrotron, CERN Yellow Reports: Monographs No. CERN-76-06, 1976.

[23] E. Forest, Beam Dynamics: A New Attitude and Framework (Hardwood Academic/CRC Press, Amsterdam, 1998).

[24] R. Tomas-Garcia, PhD Thesis, Direct measurement of resonance driving terms in the Super Proton Synchrotron (SPS) of CERN using beam position monitors, Universisty of Valencia, 2003, Report No. CERN-THESIS2003-010.

[25] B. Goddard et al., Reduction of $400 \mathrm{GeV}$ slow extraction beam loss with a wire diffuser at the SPS [Phys. Rev. Accel. Beams (to be published)].

[26] M. A. Fraser et al., Slow extraction efficiency measurements at the CERN SPS, in Proc. of IPAC'18, Vancouver, $B C$, Canada (JACoW Publishing, Geneva, Switzerland, 2018), paper TUPAF054, pp. 834-837.

[27] M. A. Fraser, L. S. Stoel, B. Goddard, V. Kain, and F. M. Velott, MD\#4164: Separatrix folding with octupoles during slow, extraction at SPS, CERN Technical Report No. CERN-ACC-NOTE-2019-0004, 2019. 